\title{
Enumeration, orthogonality and completeness of the incompressible Coriolis modes in a sphere
}

\author{
Journal Article \\ Author(s): \\ Ivers, David J.; Jackson, Andrew; Winch, Denis \\ Publication date: \\ 2015-03 \\ Permanent link: \\ https://doi.org/10.3929/ethz-b-000099633
}

Rights / license:

In Copyright - Non-Commercial Use Permitted

Originally published in:

Journal of Fluid Mechanics 766, https://doi.org/10.1017/jfm.2015.27 


\title{
Enumeration, orthogonality and completeness of the incompressible Coriolis modes in a sphere
}

\author{
D. J. Ivers ${ }^{1, \dagger}$, A. Jackson ${ }^{2}$ and D. Winch ${ }^{1}$ \\ ${ }^{1}$ School of Mathematics and Statistics, University of Sydney, NSW 2006, Australia \\ ${ }^{2}$ Institut für Geophysik, ETH, Sonneggstrasse 5, 8092 Zürich, Switzerland
}

(Received 24 June 2014; revised 28 October 2014; accepted 10 January 2015; first published online 4 February 2015)

\begin{abstract}
We consider incompressible flows in the rapid-rotation limit of small Rossby number and vanishing Ekman number, in a bounded volume with a rigid impenetrable rotating boundary. Physically the flows are inviscid, almost rigid rotations. We interpret the Coriolis force, modified by a pressure gradient, as a linear operator acting on smooth inviscid incompressible flows in the volume. The eigenfunctions of the Coriolis operator $\mathcal{C}$ so defined are the inertial modes (including any Rossby modes) and geostrophic modes of the rotating volume. We show $\mathcal{C}$ is a bounded operator and that $-\mathrm{i} \mathcal{C}$ is symmetric, so that the Coriolis modes of different frequencies are orthogonal. We prove that the space of incompressible polynomial flows of degree $N$ or less in a sphere is invariant under $\mathcal{C}$. The symmetry of $-\mathrm{i} \mathcal{C}$ thus implies the Coriolis operator is non-defective on the finite-dimensional space of spherical polynomial flows. This enables us to enumerate the Coriolis modes, and to establish their completeness using the Weierstrass polynomial approximation theorem. The fundamental tool, which is required to establish invariance of spherical polynomial flows under $\mathcal{C}$ and completeness, is that the solution of the polynomial Poisson-Neumann problem, i.e. Poisson's equation with a Neumann boundary condition and polynomial data, in a sphere is a polynomial. We also enumerate the Coriolis modes in a sphere, with careful consideration of the geostrophic modes, directly from the known analytic solutions.
\end{abstract}

Key words: rotating flows, waves in rotating fluids

\section{Introduction}

We consider the flow of a fluid, which fills a volume $V$ with a rigid closed boundary $\partial V$ and outward normal $\boldsymbol{n}$. If the fluid is non-rotating, homogeneous and inviscid, it cannot support incompressible wave motions, even in a gravitational field. For, if the velocity $v$ is irrotational, then there is a potential $\Phi$ such that $v=\nabla \Phi$ and $\nabla^{2} \Phi=\nabla \cdot \boldsymbol{v}=0$ in $V$ with $\partial_{n} \Phi=\boldsymbol{n} \cdot \boldsymbol{v}=0$ on $\partial V$. These equations imply that $\Phi$ is constant and $\boldsymbol{v}=\mathbf{0}$. However, if the fluid is rotating, it can support wave motions. Thomson (1877, p. 297) rotated a spherical shell filled with liquid and mounted on gimbals. The spherical copper shell ran smoothly even at high rotation rates. An oblate spheroid also ran smoothly, but a prolate spheroid reacted violently, destroying

$\dagger$ Email address for correspondence: david.ivers@sydney.edu.au 
the gimbal mounting through resonance of the container with internal waves in the fluid. Such free oscillations, which depend on the dynamically dominant rotation and are almost rigid rotations, are usually referred to as inertial waves, but other terms including Poincaré waves, Rossby waves or gyroscopic waves are also used. There may also be steady geostrophic modes. Our general interest is in the spatial modes of free oscillations and in the effects of forcing, when rotation is dynamically dominant. The specific aim of the present paper is to enumerate the spatial modes of inertial waves and the geostrophic modes if $V$ is a sphere, and to establish their orthogonality and completeness.

Let $\boldsymbol{v}^{\prime}$ be the fluid velocity in an inertial reference frame $O^{\prime}$. The velocity $\boldsymbol{v}^{\prime}$ is an almost rigid rotation if there exists an angular velocity $\boldsymbol{\Omega}$ such that $\max _{V} \mid \boldsymbol{v}^{\prime}-$ $\boldsymbol{\Omega} \times \boldsymbol{r}\left|\ll \max _{V}\right| \boldsymbol{v}^{\prime} \mid$. There is no unique method to determine $\boldsymbol{\Omega}$ from $\boldsymbol{v}^{\prime}$. Let $O$ be a reference frame rotating with the undetermined angular velocity $\boldsymbol{\Omega}$, with $O^{\prime}$ and $O$ sharing a common origin so that $\boldsymbol{r}^{\prime}=\boldsymbol{r}$. Let $R_{1}^{2}$ be the ratio of the kinetic energies of the flow in the two frames $O$ and $O^{\prime}$, i.e. $R_{1}^{2}:=K / K^{\prime}$, where $2 K:=\int_{V} \rho\left(\boldsymbol{v}^{\prime}-\boldsymbol{\Omega} \times \boldsymbol{r}\right)^{2} \mathrm{~d} V$ and $2 K^{\prime}:=\int_{V} \rho \boldsymbol{v}^{\prime 2} \mathrm{~d} V$. Minimisation of $R_{1}^{2}$ yields the angular velocity $\boldsymbol{\Omega}_{1}:=\boldsymbol{I}_{V}^{-1} \cdot \boldsymbol{L}_{V}$, where $\boldsymbol{I}_{V}:=\int_{V} \rho\left(r^{2} \boldsymbol{I}-\boldsymbol{r r}\right) \mathrm{d} V$ and $\boldsymbol{L}_{V}:=\int_{V} \rho \boldsymbol{r} \times \boldsymbol{v}^{\prime} \mathrm{d} V$ are the inertia tensor and the angular momentum of the fluid in $O^{\prime}$. Minimisation of $R_{2}^{2}:=K / K_{\Omega}$, where $2 K_{\Omega}:=$ $\int_{V} \rho(\boldsymbol{\Omega} \times \boldsymbol{r})^{2} \mathrm{~d} V$, yields the alternative angular velocity, $\boldsymbol{\Omega}_{2}=\boldsymbol{\Omega}_{1}\left(2 K^{\prime} / \boldsymbol{L}_{V} \cdot \boldsymbol{\Omega}_{1}\right)$. The two values $\boldsymbol{\Omega}_{1}$ and $\boldsymbol{\Omega}_{2}$ differ only slightly, if $\min R_{1}=\min R_{2} \ll 1$. We will consider flows with the same rigidly rotating base.

We non-dimensionalise $t, \boldsymbol{\Omega}, \boldsymbol{r}, \boldsymbol{v}$ and $\rho$ on the rotation time scale $\left(2 \Omega_{\star}\right)^{-1}$ with a typical length $L_{\star}$, rotation rate $\Omega_{\star}$, speed $v_{\star}$ and density $\rho_{\star}$. Thus the velocities are related by $\boldsymbol{v}^{\prime}=\operatorname{Rov}+\boldsymbol{\Omega} \times \boldsymbol{r}$, scaling $\boldsymbol{v}^{\prime}$ with a typical speed $\Omega_{\star} L_{\star}$ relative to $O^{\prime}$ and introducing the Rossby number $R o:=v_{\star} / \Omega_{\star} L_{\star} \approx \min \left(R_{1}, R_{2}\right)$. The description in the rotating frame is simpler than the inertial frame $O^{\prime}$ for flows that are almost rigid rotations. Under the transformation from $O^{\prime}$ to $O$, a vector field $\boldsymbol{F}^{\prime}$ defined independently of the reference frame maps to $\boldsymbol{F}=\boldsymbol{F}^{\prime}$ and the spatial gradient is invariant, $\nabla^{\prime} \boldsymbol{F}^{\prime}=\boldsymbol{\nabla} \boldsymbol{F}$. This is false for frame-dependent (relative) fields such as the velocity, thus

$$
R o^{-1} \boldsymbol{v}^{\prime} \cdot \nabla^{\prime} \boldsymbol{v}^{\prime}=R o v \cdot \nabla \boldsymbol{v}+\boldsymbol{\Omega} \times \boldsymbol{v}+R^{-1} \boldsymbol{\Omega} \times(\boldsymbol{\Omega} \times \boldsymbol{r})+\boldsymbol{\Omega} \times \boldsymbol{r} \cdot \boldsymbol{\nabla} \boldsymbol{v} .
$$

Thus $\boldsymbol{v}^{\prime} \cdot \nabla^{\prime} \boldsymbol{v}^{\prime}$ in the inertial frame cannot be neglected. Combined with $2 R o^{-1} \partial_{t}^{\prime} \boldsymbol{v}^{\prime}=$ $2 \partial_{t} \boldsymbol{v}+\boldsymbol{\Omega} \times \boldsymbol{v}+2$ Po $\dot{\boldsymbol{\Omega}} \times \boldsymbol{r}-\boldsymbol{\Omega} \times \boldsymbol{r} \cdot \boldsymbol{\nabla} \boldsymbol{v}$, this gives the transformation for the convective derivative,

$$
R o^{-1} D_{t}^{\prime} \boldsymbol{v}^{\prime}=D_{t} \boldsymbol{v}+\boldsymbol{\Omega} \times \boldsymbol{v}+P o \dot{\boldsymbol{\Omega}} \times \boldsymbol{r}+\frac{1}{2} R o^{-1} \boldsymbol{\Omega} \times(\boldsymbol{\Omega} \times \boldsymbol{r})
$$

where $D_{t} \boldsymbol{v}:=\partial_{t} \boldsymbol{v}+(\operatorname{Ro} \boldsymbol{v} \cdot \nabla \boldsymbol{v}) / 2, D_{t}^{\prime} \boldsymbol{v}^{\prime}:=\partial_{t}^{\prime} \boldsymbol{v}^{\prime}+\left(\boldsymbol{v}^{\prime} \cdot \nabla^{\prime} \boldsymbol{v}^{\prime}\right) / 2$ and Po is the Poincaré number. Thus $R o \rightarrow 0$ does not remove the advective term in the inertial frame.

Thus the effects of steady rotation can be characterised mathematically by the Coriolis force in a rotating reference frame. In a frame $O$ rotating with angular velocity $\boldsymbol{\Omega}$ the velocity $\boldsymbol{v}$ is governed by the momentum equation,

$$
\partial_{t} \boldsymbol{v}+\boldsymbol{\Omega} \times \boldsymbol{v}+\rho^{-1} \nabla p=-\frac{1}{2} R o \boldsymbol{v} \cdot \nabla \boldsymbol{v}-\nabla U_{e}+E \nabla^{2} \boldsymbol{v}-P o \dot{\boldsymbol{\Omega}} \times \boldsymbol{r}+E l \rho^{-1} \boldsymbol{F}_{L},
$$

where $\dot{\boldsymbol{\Omega}}:=\mathrm{d} \boldsymbol{\Omega} / \mathrm{d} t, \boldsymbol{r}$ is the radius vector and $U_{e}$ is the effective gravitational potential modified by the centripetal acceleration $\boldsymbol{\Omega} \times(\boldsymbol{\Omega} \times \boldsymbol{r})$. If the fluid is electrically conducting there is also the Lorentz force $\boldsymbol{F}_{L}$. The pressure has been 
non-dimensionalised with a typical pressure $\rho_{\star} 4 \Omega_{\star}^{2} L_{\star}$. The dimensionless rotation rate $\boldsymbol{\Omega}$ may be time-dependent with only $|\boldsymbol{\Omega}| \approx 1$; if $\boldsymbol{\Omega}$ is constant, then $\boldsymbol{\Omega}$ is a unit vector, which will be denoted $\widehat{\boldsymbol{\Omega}}$. The dimensionless Rossby, Ekman, Poincaré and Elsasser numbers, $R o, E:=v / 2 \Omega_{\star} L_{\star}^{2}, P o$ and $E l$, are typical magnitude ratios of the advective, viscous, Poincaré and Lorentz forces to the Coriolis force. The factor $(1 / 2)$ in (1.3) is due to the definition of the Rossby number and arises from the time scale. We are interested in the rapid-rotation regime $R o \ll 1$ and $E \ll 1$. The limit $E \rightarrow 0$ removes the viscous term in an inertial frame, since $\nabla^{\prime} \boldsymbol{v}^{\prime}+\left(\nabla^{\prime} \boldsymbol{v}^{\prime}\right)^{\mathrm{T}}=\boldsymbol{\nabla} \boldsymbol{v}+(\boldsymbol{\nabla} \boldsymbol{v})^{\mathrm{T}}$ and $\nabla^{\prime} \cdot \boldsymbol{v}^{\prime}=\boldsymbol{\nabla} \cdot \boldsymbol{v}$ imply $E\left(\nabla^{\prime}\right)^{2} \boldsymbol{v}^{\prime}=E R o \nabla^{2} \boldsymbol{v}$. In the non-magnetic rapid-rotation limit $E l, R o, E, P o \rightarrow 0$, with constant angular velocity and uniform density $(\rho=1)$, the momentum equation (1.3) and its solution $\boldsymbol{v}(\boldsymbol{r}, t ; R o, E, P o, E l)$ reduce on setting $E l=R o=E=P o=0$ to

$$
\partial_{t} \boldsymbol{v}+\boldsymbol{\Omega} \times \boldsymbol{v}=-\nabla P,
$$

where the velocity is $\boldsymbol{v}(\boldsymbol{r}, t ; 0,0,0,0)$ and $P:=p+U_{e}$. We do not consider in the present work if the velocity $\boldsymbol{v}(\boldsymbol{r}, t ; R o, E, P o, E l) \rightarrow \boldsymbol{v}(\boldsymbol{r}, t ; 0,0,0,0)$ as $R o, E, P o, E l \rightarrow 0$.

We assume $\partial V$ is impenetrable, so $\boldsymbol{n} \cdot\left(\boldsymbol{v}-\boldsymbol{v}_{\partial V}\right)=0$ on $\partial V$, where $\boldsymbol{n}$ is a (possibly non-unit) normal on $\partial V$. We further assume that $\partial V$ rotates with a constant angular velocity, which is consistent with the almost rigid rotation flows. We do not consider free surfaces or impulsive motion of $\partial V$ (e.g. see Bryan 1889 and Hough 1895). Henceforth, $O$ is the boundary frame, $\boldsymbol{n}$ is steady and the boundary condition reduces to

$$
\boldsymbol{n} \cdot \boldsymbol{v}=0 \quad \text { on } \partial V .
$$

We assume the flow is incompressible,

$$
\nabla \cdot v=0 \text { in } V
$$

except for a brief discussion in the final section $\S 9$.

Mathematically the inertial wave problem consists of (1.4)-(1.6). Analytic solutions are usually derived from the formulation in terms of the pressure $P$ outlined in $\S 2$. The velocity can be eliminated, yielding the free Poincaré equation,

$$
\partial_{t}^{2} \nabla^{2} P+(\widehat{\boldsymbol{\Omega}} \cdot \nabla)^{2} P=0 .
$$

Thomson (1880) determined the solutions for inertial waves in a cylinder and cylindrical shell rotating about the cylindrical axis. Poincaré (1885a, pp. 355-365) (not to be confused with Poincare $(1885 b, c)$ of the same title), in a study of the stability of a fluid ellipsoid with a free surface, gave an incomplete derivation of the inertial modes for an ellipsoid rotating about a principal axis in terms of Lamé functions; see also Hough (1895, Appendix p. 479). Bryan (1889) gave a full derivation of the inertial modes in terms of spheroidal harmonics for a spheroid rotating about its symmetry axis; see also Kudlick (1966). Taylor (1922), Rao (1966) and Nurijanyan, Bokhove \& Maas (2013) computed the inertial modes for a rectangular prism rotating about a centred axis perpendicular to a face. Rieutord (1991) and Rieutord \& Valdettaro (1997) computed the viscous inertial modes for a spherical shell with no-slip and stress-free boundary conditions. Solutions for the periodic plane layer and the periodic duct rotating about a centred axis perpendicular to impenetrable plane boundaries are well known. Pedlosky \& Greenspan (1967) derived approximate solutions for the sliced cylinder $\left\{(x, y, z) \in E^{3} \mid x^{2}+y^{2}<1\right.$, ay $\left.<z<b\right\}$, where $E^{3}$ is 
Euclidean space with a set of Cartesian coordinates $(x, y, z)$ and $a, b$ are constants. Inertial waves underlie many geophysical and astrophysical phenomena (see Zhang 1993; Cui, Zhang \& Liao 2014).

To study the spatial modes of inertial waves we consider the steady incompressible Coriolis operator $\mathcal{C}$ defined by

$$
\mathcal{C} v:=\widehat{\boldsymbol{\Omega}} \times \boldsymbol{v}+\nabla p
$$

subject to $\boldsymbol{\nabla} \cdot \boldsymbol{v}=0$ in $V$ and $\boldsymbol{n} \cdot \boldsymbol{v}=0$ on $\partial V$, i.e. (1.5) and (1.6). The operator $\mathcal{C}$ is a time-independent linear operator. This definition of $\mathcal{C}$ is incomplete until the auxiliary function or pressure $p$ has been defined. We require that $\mathcal{C} v$ satisfies (1.6) and (1.5), i.e.

$$
\boldsymbol{\nabla} \cdot(\mathcal{C} \boldsymbol{v})=0 \quad \text { in } V, \quad \boldsymbol{n} \cdot \mathcal{C} \boldsymbol{v}=0 \quad \text { on } \partial V
$$

and hence $p$ must satisfy the elliptic Poisson-Neumann problem,

$$
\nabla^{2} p=-\nabla \cdot(\widehat{\boldsymbol{\Omega}} \times \boldsymbol{v}) \quad \text { in } V ; \quad \partial_{n} p=-\boldsymbol{n} \cdot \widehat{\boldsymbol{\Omega}} \times \boldsymbol{v} \quad \text { on } \partial V
$$

where $v$ is prescribed. This associates to each $v$ a $p$ which is unique, if the condition $\int_{V} p \mathrm{~d} V=0$ is imposed. We give an explicit expression for $\mathcal{C} \boldsymbol{v}$ in terms of $\boldsymbol{v}$ in $\S 3$.

It is useful to formulate the problem in terms of the space of functions $v$ on which $\mathcal{C}$ acts. Let $\mathcal{V}^{k}(V)$, where $k$ is a positive integer, be the space of $C^{k}$ (k-times continuously differentiable) inviscid solenoidal velocities in $V$, i.e. which satisfy (1.5) and (1.6). Thus $\mathcal{V}^{k}(V):=\left\{\boldsymbol{v} \in\left[C^{k}(V)\right]^{3} \mid \boldsymbol{\nabla} \cdot \boldsymbol{v}=0\right.$ in $V, \boldsymbol{n} \cdot \boldsymbol{v}=0$ on $\left.\partial V\right\}$ with the inner product

$$
\left(\boldsymbol{v}_{1}, \boldsymbol{v}_{2}\right):=\int_{V} \boldsymbol{v}_{1} \cdot \boldsymbol{v}_{2}^{*} \mathrm{~d} V,
$$

where the asterisk denotes complex conjugation and $C^{k}(V)$ is the space of complexvalued $C^{k}$ scalar functions on $V$. The associated norm is $\|\boldsymbol{v}\|=(\boldsymbol{v}, \boldsymbol{v})^{1 / 2},\left(\boldsymbol{v}_{1}, \boldsymbol{v}_{2}\right)=$ $\left(\boldsymbol{v}_{2}, \boldsymbol{v}_{1}\right)^{*}$ and the Cauchy-Schwartz inequality $\left|\left(\boldsymbol{v}_{1}, \boldsymbol{v}_{2}\right)\right| \leqslant\left\|\boldsymbol{v}_{1}\right\|\left\|\boldsymbol{v}_{2}\right\|$ holds. The flows have finite kinetic energy. Thus we require that the linear operator $\mathcal{C}$ maps flows in $\mathcal{V}^{1}(V)$ into $\mathcal{V}^{1}(V)$. The space $\mathcal{V}^{1}(V)$ is not complete and therefore not a Hilbert space; but see the discussion in $\S 9$. This does not limit the present results since we do not need to prove the general existence of solutions. Instead we consider restrictions of $\mathcal{C}$ to finite-dimensional spaces of polynomial flows and use the Weierstrass theorem on uniform polynomial approximation of continuous functions in a bounded region to approximate flows in $\mathcal{V}^{1}(V)$. We also introduce the associated space of pressures on $V, \mathcal{P}^{k}(V):=\left\{p \in C^{k}(V) \mid \int_{V} p \mathrm{~d} V=0\right\}$, with inner product

$$
\left(p_{1}, p_{2}\right):=\int_{V} \nabla p_{1} \cdot \nabla p_{2}^{*} \mathrm{~d} V .
$$

A second useful operator associated with the Navier-Stokes equation (1.3) is the Stokes operator,

$$
\mathcal{S} v:=-\nabla^{2} v+\nabla p
$$

with $\boldsymbol{\nabla} \cdot \boldsymbol{v}=0$ in $V, \boldsymbol{n} \cdot \boldsymbol{v}=0$ on $\partial V$, additionally subject to either the no-slip condition $\boldsymbol{n} \times \boldsymbol{v}=\mathbf{0}$ or the stress-free condition $\boldsymbol{n} \cdot\left[\boldsymbol{\nabla} \boldsymbol{v}+(\boldsymbol{\nabla} \boldsymbol{v})^{\mathrm{T}}\right] \times \boldsymbol{n}=\mathbf{0}$ on $\partial V . \mathcal{S}$ maps a flow $\boldsymbol{v} \in \mathcal{V}^{2}(V)$ into $\mathcal{V}^{2}(V)$. Here $p$ must satisfy the Poisson-Neumann problem,

$$
\nabla^{2} p=0 \quad \text { in } V ; \quad \partial_{n} p=\boldsymbol{n} \cdot \nabla^{2} \boldsymbol{v} \quad \text { on } \partial V .
$$


In terms of the operators $\mathcal{C}$ and $\mathcal{S}$ with associated pressures $p_{C}$ and $p_{S}$, the NavierStokes equation (1.3) with uniform density or in the Boussinesq approximation can be written as

$$
\partial_{t} \boldsymbol{v}+\mathcal{C} \boldsymbol{v}+E \mathcal{S} \boldsymbol{v}=-\nabla\left(p-p_{C}-p_{S}\right)+P o \boldsymbol{F}_{P}+\frac{1}{2} R o \boldsymbol{F}_{a}+E l \boldsymbol{F}_{L}+\boldsymbol{F}_{g},
$$

where $\boldsymbol{F}_{P}, \boldsymbol{F}_{a}, \boldsymbol{F}_{L}, \boldsymbol{F}_{g}$ are the Poincaré, advective, Lorentz and gravitational or buoyancy force. The forces $\boldsymbol{F}_{P}, \boldsymbol{F}_{a}, \boldsymbol{F}_{L}, \boldsymbol{F}_{g}$ can be projected individually onto $\mathcal{V}^{k}(V)$ by subtracting a pressure gradient: $\boldsymbol{F}=\boldsymbol{f}+\nabla p_{F}$ and $p_{F}$ is chosen so that $\boldsymbol{f} \in \mathcal{V}^{k}(V)$, i.e.

$$
\nabla \cdot \boldsymbol{f}=0 \quad \text { in } V, \quad \boldsymbol{n} \cdot \boldsymbol{f}=0 \quad \text { on } \partial V .
$$

Thus the pressure $p_{F}$ is the solution of the Poisson-Neumann problem,

$$
\nabla^{2} p_{F}=-\nabla \cdot \boldsymbol{F} \quad \text { in } V ; \quad \partial_{n} p_{F}=-\boldsymbol{n} \cdot \boldsymbol{F} \quad \text { on } \partial V ; \quad \int_{V} p_{F} \mathrm{~d} V=0 . \quad(1.17 a-c)
$$

Observe that this problem, and the specific cases (1.10) and (1.15), are self-consistent. Hence projecting $\boldsymbol{F}_{P}, \boldsymbol{F}_{a}, \boldsymbol{F}_{L}, \boldsymbol{F}_{g}$ yields $\boldsymbol{f}_{P}, \boldsymbol{f}_{a}, \boldsymbol{f}_{L}, \boldsymbol{f}_{g} \in \mathcal{V}^{k}(V)$ with associated pressures $p_{P}, p_{a}, p_{L}, p_{g}$ satisfying (1.17). The momentum equation reduces to

$$
\partial_{t} v+\mathcal{C} v+E \mathcal{S} v=P o f_{P}+\frac{1}{2} R o f_{a}+E l f_{L}+f_{g} .
$$

The divergence of (1.15) yields $\nabla^{2}\left(p-p_{C}-E p_{S}-p_{P}-\left(R o p_{a} / 2\right)-E l p_{L}-p_{g}\right)=$ 0 . Further, since $\boldsymbol{n}$ is independent of time, taking the dot product of $\boldsymbol{n}$ with (1.16) yields $\partial_{n}\left(p-p_{C}-E p_{S}-P o p_{P}-\left(R o p_{a} / 2\right)-E l p_{L}-p_{g}\right)=0$ on the boundary $\partial V$. Hence $p-p_{C}-E p_{S}-P o p_{P}-\left(R o p_{a} / 2\right)-E l p_{L}-p_{g}=0$. Thus we consider the model forced-momentum equation in the rapid-rotation limit,

$$
\partial_{t} v+\mathcal{C} v=f
$$

where $\boldsymbol{f}$ satisfies conditions (1.16).

We consider properties of $\mathcal{C}$ for general fluid volumes $V$ in $\S 3$, in particular we show that $\mathcal{C}$ is a bounded operator on $\mathcal{V}^{1}(V)$ and $-\mathrm{i} \mathcal{C}$ is symmetric on $\mathcal{V}^{1}(V)$. Our primary interest is the discrete spectrum of $\mathcal{C}$, which consists of the eigenvalues $\lambda$ of $\mathcal{C}$, and the eigenfunctions $\boldsymbol{v}(\neq \mathbf{0})$, which satisfy $\mathcal{C} v=\lambda \boldsymbol{v}$. Throughout we associate with an eigenvalue $\lambda$ of $\mathcal{C}$ an angular frequency $\omega=-\mathrm{i} \lambda$, so that $-\mathrm{i} \mathcal{C} \boldsymbol{v}=\omega \boldsymbol{v}$. We will refer to $(\boldsymbol{v}, \lambda)$ as a (discrete) Coriolis mode. Physically, Coriolis modes are geostrophic modes or the spatial modes of inertial waves, or more generally Rossby waves, e.g. in a sliced cylinder (Pedlosky \& Greenspan 1967). We will refer to Coriolis modes of zero and non-zero frequency as geostrophic and inertial modes respectively. In $\S 4$ we enumerate by construction the Coriolis velocity modes $\boldsymbol{v}_{n, j}^{m}$ in a sphere. The index $n$ is the polynomial degree of the velocity mode, $m$ is the azimuthal wavenumber and $j$ labels the frequency. For the zero frequency, an infinite orthogonal set of geostrophic modes must be constructed, since it is infinitely degenerate.

In $\S 5$ we determine the dimension of the space of polynomial flows in a sphere. This together with Theorem 6.4 provides an alternative method for enumerating the Coriolis modes which avoids explicit construction of the modes. In $\S 6$ we show in Theorem 6.3 that the Coriolis operator is well defined on the space of polynomial flows in a sphere. Theorem 6.4 follows from this. This is based on the fundamental tool, Theorem 6.2, that the solution in a sphere of the polynomial Poisson-Neumann 
problem, i.e. Poisson's equation with a Neumann boundary condition and polynomial data, is polynomial.

Consider the integral

$$
I_{n_{1}, j_{1}, n_{2}, j_{2}}^{m_{1}, m_{2}}:=\left(\nabla^{2} \boldsymbol{v}_{n_{1}, j_{1}}^{m_{1}}, \boldsymbol{v}_{n_{2}, j_{2}}^{m_{2}}\right) .
$$

Zhang et al. (2001) derived a representation for $\boldsymbol{v}_{n, j}^{m}$ in terms of the monomial basis $\left\{s^{i} z^{j} \mathrm{e}^{\mathrm{i} m \phi}\right\}$ and showed that $I_{n, j, n, j}^{m, m}=0$ for a sphere. Zhang, Liao \& Earnshaw (2004) extended this result to a spheroid rotating about its axis. Liao \& Zhang (2009) showed that $I_{n_{1}, j_{1}, n_{2}, j_{2}}^{m, m}=0$ for a sphere if $n_{1} \leqslant n_{2}$. A proof of this directly from the governing equations remains elusive. Indeed, these remarkable results were our original motivation for studying the polynomial properties of inertial modes and the Coriolis operator. It is shown in $\S 7$ that $I_{n_{1}, j_{1}, n_{2}, j_{2}}^{m, m}=0$ for a sphere if $n_{1} \leqslant n_{2}+1$, as a simple corollary of the results in $\S 6$.

In $\$ 8$ we again use Theorem 6.2 to establish the most important result of the paper, namely Theorem 8.1, that the Coriolis modes in a sphere $V$ are complete. Completeness of inertial modes has been established for a periodic duct (Cui et al. 2014).

In the concluding $\S 9$ we outline an abstract existence theorem for Coriolis modes in a general bounded fluid volume. We also briefly discuss applications and the generalisations of the important time-dependent incompressible Coriolis operator and the anelastic Coriolis operator.

\section{Poincaré's equation}

Since $\boldsymbol{\Omega}$ is constant in time, the velocity $\boldsymbol{v}$ can be eliminated from (1.19) and (1.6) as follows. The cross product of (1.19) with $\widehat{\boldsymbol{\Omega}}$, noting (1.8), gives $\partial_{t}(\widehat{\boldsymbol{\Omega}} \times \boldsymbol{v})+(\widehat{\boldsymbol{\Omega}}$. $\boldsymbol{v}) \widehat{\boldsymbol{\Omega}}-\boldsymbol{v}=-\widehat{\boldsymbol{\Omega}} \times(\nabla p-\boldsymbol{f})$. Substituting $\widehat{\boldsymbol{\Omega}} \times \boldsymbol{v}=-(\nabla p-\boldsymbol{f})-\partial_{t} \boldsymbol{v}$,

$$
\partial_{t}^{2} \boldsymbol{v}+\boldsymbol{v}=-\partial_{t}(\nabla p-\boldsymbol{f})+\widehat{\boldsymbol{\Omega}} \times(\nabla p-\boldsymbol{f})+(\widehat{\boldsymbol{\Omega}} \cdot \boldsymbol{v}) \widehat{\boldsymbol{\Omega}} .
$$

The dot product of (1.19) with $\widehat{\boldsymbol{\Omega}}$ gives

$$
\partial_{t}(\widehat{\boldsymbol{\Omega}} \cdot \boldsymbol{v})=-\widehat{\boldsymbol{\Omega}} \cdot(\nabla p-\boldsymbol{f}) .
$$

Differentiating (2.1) with respect to $t$ and using the previous equation (2.2) to eliminate $\partial_{t}(\widehat{\boldsymbol{\Omega}} \cdot \boldsymbol{v})$ from the right-hand side,

$$
\partial_{t}^{3} \boldsymbol{v}+\partial_{t} \boldsymbol{v}=-\partial_{t}^{2}(\nabla p-\boldsymbol{f})+\widehat{\boldsymbol{\Omega}} \times \partial_{t}(\nabla p-\boldsymbol{f})-\widehat{\boldsymbol{\Omega}} \cdot(\nabla p-\boldsymbol{f}) \widehat{\boldsymbol{\Omega}} .
$$

The divergence of (2.3) yields the forced Poincaré equation,

$$
\partial_{t}^{2} \nabla^{2} p+(\widehat{\boldsymbol{\Omega}} \cdot \nabla)^{2} p=\widehat{\boldsymbol{\Omega}} \cdot \boldsymbol{\nabla} \times \partial_{t} \boldsymbol{f}+\widehat{\boldsymbol{\Omega}} \cdot \boldsymbol{\nabla}(\widehat{\boldsymbol{\Omega}} \cdot \boldsymbol{f}),
$$

noting $\nabla \cdot f=0$. Since the boundary $\partial V$ and $\boldsymbol{n}$ are steady in the rotating frame $O$, the dot product of (2.3) with $\boldsymbol{n}$ yields the boundary condition,

$$
\partial_{t}^{2} \partial_{n} p-\boldsymbol{n} \times \widehat{\boldsymbol{\Omega}} \cdot \partial_{t} \nabla p+(\widehat{\boldsymbol{\Omega}} \cdot \nabla p) \boldsymbol{n} \cdot \widehat{\boldsymbol{\Omega}}=-\boldsymbol{n} \cdot \widehat{\boldsymbol{\Omega}} \times \partial_{t} \boldsymbol{f}+\widehat{\boldsymbol{\Omega}} \cdot \boldsymbol{f} \boldsymbol{n} \cdot \widehat{\boldsymbol{\Omega}},
$$

noting $\boldsymbol{n} \cdot \boldsymbol{f}=0$. 
The initial value problems consisting of (1.19), subject to (1.6) and (1.5), or (2.4), subject to (2.5), with $\boldsymbol{v}$ or $p$ prescribed at $t=0$ can be solved in principle using Laplace transforms in $t$. Alternatively, there is the modal approach adopted here of separating the time dependence. Thus, if $\boldsymbol{f}(\boldsymbol{r}, t)=\boldsymbol{f}(\boldsymbol{r}) \mathrm{e}^{-\lambda t}$, then (1.19) has a forced solution of the form $\boldsymbol{v}(\boldsymbol{r}, t)=\boldsymbol{v}(\boldsymbol{r}) \mathrm{e}^{-\lambda t}$ with $p(\boldsymbol{r}, t)=p(\boldsymbol{r}) \mathrm{e}^{-\lambda t}$. The time dependence can also be expressed in terms of the angular frequency $\omega=-\mathrm{i} \lambda$ introduced in the introduction. The momentum equation (2.1) and Poincaré's equation (2.4) reduce to

$$
\begin{gathered}
-\lambda \boldsymbol{v}+\widehat{\boldsymbol{\Omega}} \times \boldsymbol{v}=-\nabla p+\boldsymbol{f} \\
\lambda^{2} \nabla^{2} p+(\widehat{\boldsymbol{\Omega}} \cdot \nabla)^{2} p=-\lambda \widehat{\boldsymbol{\Omega}} \cdot \nabla \times \boldsymbol{f}+\widehat{\boldsymbol{\Omega}} \cdot \nabla(\widehat{\boldsymbol{\Omega}} \cdot \boldsymbol{f}) .
\end{gathered}
$$

From (2.3), or using the fact that $a \boldsymbol{x}+\boldsymbol{b} \times \boldsymbol{x}=\boldsymbol{c}$ implies $\left(a^{2}+b^{2}\right) \boldsymbol{x}=a \boldsymbol{c}-\boldsymbol{b} \times \boldsymbol{c}+(\boldsymbol{b}$. $\boldsymbol{c} / a) \boldsymbol{b}$ in (2.7),

$$
\lambda\left(1+\lambda^{2}\right) \boldsymbol{v}=\lambda^{2}(\nabla p-\boldsymbol{f})+\lambda \widehat{\boldsymbol{\Omega}} \times(\nabla p-\boldsymbol{f})+\widehat{\boldsymbol{\Omega}} \cdot(\nabla p-\boldsymbol{f}) \widehat{\boldsymbol{\Omega}} .
$$

The boundary condition (2.4) becomes

$$
\lambda^{2} \partial_{n} p+\lambda \boldsymbol{n} \times \widehat{\boldsymbol{\Omega}} \cdot \nabla p+(\widehat{\boldsymbol{\Omega}} \cdot \nabla p) \boldsymbol{n} \cdot \widehat{\boldsymbol{\Omega}}=\lambda \boldsymbol{n} \cdot \widehat{\boldsymbol{\Omega}} \times \boldsymbol{f}+\widehat{\boldsymbol{\Omega}} \cdot \boldsymbol{f} \boldsymbol{n} \cdot \widehat{\boldsymbol{\Omega}}
$$

The forced Poincaré equation (2.7) in $V$ must be solved subject to this condition on $\partial V$.

For real frequencies $\omega,(2.7)$ is elliptic if $|\omega| \geqslant 1$ and hyperbolic if $|\omega|<1$. The characteristics of (2.7) are the cones $z= \pm \omega s / \sqrt{1-\omega^{2}}$, which are real in the hyperbolic case. The kinetic energy of the mode $\{\boldsymbol{v}, \omega\}$ is $(\boldsymbol{v}, \boldsymbol{v}) / 2$, which is constant since $\left(\boldsymbol{v} \mathrm{e}^{-\mathrm{i} \omega t}, \boldsymbol{v} \mathrm{e}^{-\mathrm{i} \omega t}\right)=(\boldsymbol{v}, \boldsymbol{v})$. If $V$ is axisymmetric, $\widehat{\boldsymbol{\Omega}}$ and the $z$-axis are co-axial with the symmetry axis, then the solutions decouple into azimuthal modes $P(\boldsymbol{r}) \propto \mathrm{e}^{\mathrm{i} m \phi}$, $m \in \mathbb{Z}$ and $P(\boldsymbol{r}, t) \propto \mathrm{e}^{\mathrm{i}(m \phi-\omega t)}$. Thus we adopt the convention that $\omega>0$ corresponds to eastward (westward) propagation for positive (negative) azimuthal wavenumbers $m>0(m<0)$.

\section{The Coriolis operator}

In this section we prove some general results for the Coriolis operator $\mathcal{C}$ acting on the space $\mathcal{V}^{1}(V)$ for a general bounded fluid region $V$, not just for a sphere. Given $\boldsymbol{v} \in$ $\mathcal{V}^{1}(V)$, then $\mathcal{C} v \in \mathcal{V}^{1}(V)$ if the pressure $p$ is determined from the Poisson-Neumann problem (1.10). This elliptic problem is well posed, unlike the ill-posed hyperbolic problem, equations (2.7) and (2.9) for real $-1<\omega<1$, which determines the pressure. The infinite-dimensional operator $\mathcal{C}$ is linear, non-local and can be expressed explicitly in terms of $v$,

$$
\mathcal{C} \boldsymbol{v}=\widehat{\boldsymbol{\Omega}} \times \boldsymbol{v}+\int_{V}\left[\nabla \nabla^{\prime} G\left(\boldsymbol{r}^{\prime}, \boldsymbol{r}\right)\right] \cdot\left(\widehat{\boldsymbol{\Omega}} \times \boldsymbol{v}^{\prime}\right) \mathrm{d} V^{\prime}
$$

where $G$ is the Green's function of the Poisson-Neumann problem, $\nabla^{2} G=\delta\left(\boldsymbol{r}-\boldsymbol{r}^{\prime}\right)$ in $V$ with $\partial_{n} G=0$ on $\partial V$. Equation (3.1) can be derived using Green's representation formula,

$$
p\left(\boldsymbol{r}^{\prime}\right)=-\int_{V} \frac{\nabla^{2} p}{4 \pi\left|\boldsymbol{r}-\boldsymbol{r}^{\prime}\right|} \mathrm{d} V-\int_{\partial V} \mathrm{~d} \boldsymbol{S} \cdot\left\{p \nabla\left(\frac{1}{4 \pi\left|\boldsymbol{r}-\boldsymbol{r}^{\prime}\right|}\right)-\frac{\nabla p}{4 \pi\left|\boldsymbol{r}-\boldsymbol{r}^{\prime}\right|}\right\}, \quad \boldsymbol{r}^{\prime} \in V .
$$


Substituting from (1.10) yields

$$
p\left(\boldsymbol{r}^{\prime}\right)=\int_{V} \frac{\nabla \cdot(\widehat{\boldsymbol{\Omega}} \times \boldsymbol{v})}{4 \pi\left|\boldsymbol{r}-\boldsymbol{r}^{\prime}\right|} \mathrm{d} V-\int_{\partial V} \mathrm{~d} \boldsymbol{S} \cdot\left\{p \nabla\left(\frac{1}{4 \pi\left|\boldsymbol{r}-\boldsymbol{r}^{\prime}\right|}\right)+\frac{\widehat{\boldsymbol{\Omega}} \times \boldsymbol{v}}{4 \pi\left|\boldsymbol{r}-\boldsymbol{r}^{\prime}\right|}\right\} .
$$

To eliminate $p$ from the surface integral choose a harmonic function $h\left(\boldsymbol{r}, \boldsymbol{r}^{\prime}\right)$ such that

$$
\nabla^{2} h\left(\boldsymbol{r}, \boldsymbol{r}^{\prime}\right)=0 \quad \text { in } V, \quad \boldsymbol{n} \cdot \nabla h\left(\boldsymbol{r}, \boldsymbol{r}^{\prime}\right)=-\boldsymbol{n} \cdot \nabla\left(4 \pi\left|\boldsymbol{r}-\boldsymbol{r}^{\prime}\right|\right)^{-1} \quad \text { on } \partial V .
$$

Then, by Green's first theorem in $p$ and $h$,

$$
\int_{V} h\left(\boldsymbol{r}, \boldsymbol{r}^{\prime}\right) \nabla \cdot(\widehat{\boldsymbol{\Omega}} \times \boldsymbol{v}) \mathrm{d} V-\int_{\partial V}\left[p \nabla h\left(\boldsymbol{r}, \boldsymbol{r}^{\prime}\right)+h\left(\boldsymbol{r}, \boldsymbol{r}^{\prime}\right) \widehat{\boldsymbol{\Omega}} \times \boldsymbol{v}\right] \cdot \mathrm{d} \boldsymbol{S}=0 .
$$

Combining the last two integral equations,

$$
p\left(\boldsymbol{r}^{\prime}\right)=-\int_{V} G\left(\boldsymbol{r}, \boldsymbol{r}^{\prime}\right) \nabla \cdot(\widehat{\boldsymbol{\Omega}} \times \boldsymbol{v}) \mathrm{d} V+\int_{\partial V} G\left(\boldsymbol{r}, \boldsymbol{r}^{\prime}\right) \widehat{\boldsymbol{\Omega}} \times \boldsymbol{v} \cdot \mathrm{d} \boldsymbol{S},
$$

where $G\left(\boldsymbol{r}, \boldsymbol{r}^{\prime}\right):=-h\left(\boldsymbol{r}, \boldsymbol{r}^{\prime}\right)-\left(4 \pi\left|\boldsymbol{r}-\boldsymbol{r}^{\prime}\right|\right)^{-1}$ is the (interior) Neumann Green's function for $V$. Integrating by parts gives

$$
p\left(\boldsymbol{r}^{\prime}\right)=\int_{V} \nabla G\left(\boldsymbol{r}, \boldsymbol{r}^{\prime}\right) \cdot(\widehat{\boldsymbol{\Omega}} \times \boldsymbol{v}) \mathrm{d} V
$$

and interchanging $\boldsymbol{r}$ and $\boldsymbol{r}^{\prime}$ yields (3.1). For the sphere $r<a$,

$$
4 \pi G\left(\boldsymbol{r}, \boldsymbol{r}^{\prime}\right)=\frac{1}{a} \ln \left(a^{2}-\boldsymbol{r} \cdot \boldsymbol{r}^{\prime}+\left|\boldsymbol{r} r^{\prime}-a^{2} \boldsymbol{r}^{\prime} / \boldsymbol{r}^{\prime}\right|\right)-\frac{a}{\left|\boldsymbol{r} r^{\prime}-a^{2} \boldsymbol{r}^{\prime} / \boldsymbol{r}^{\prime}\right|}-\frac{1}{\left|\boldsymbol{r}-\boldsymbol{r}^{\prime}\right|} .
$$

The operator $\mathcal{C}$ is bounded (Chossat 1979) on $\mathcal{V}^{1}(V)$, since

$$
\|\mathcal{C} v\|^{2}=(\mathcal{C} v, \widehat{\boldsymbol{\Omega}} \times \boldsymbol{v})+(\mathcal{C} v, \nabla p)=\|\mathcal{C} \boldsymbol{v}\|\|\widehat{\boldsymbol{\Omega}} \times \boldsymbol{v}\| \leqslant\|\mathcal{C} \boldsymbol{v}\|\|\boldsymbol{v}\|,
$$

using the Cauchy-Schwartz inequality and noting $(\mathcal{C} v, \nabla p)=0$ by the divergence theorem, $\boldsymbol{\nabla} \cdot(\mathcal{C} \boldsymbol{v})=0$ in $V$ and $\boldsymbol{n} \cdot \mathcal{C} \boldsymbol{v}=0$ on $\partial V$. Hence $\|\mathcal{C} \boldsymbol{v}\| \leqslant\|\boldsymbol{v}\|$ or, in terms of the operator norm $\|\mathcal{C}\|$ of $\mathcal{C}$ induced by the vector norm $\|\boldsymbol{v}\|$,

$$
\|\mathcal{C}\|:=\sup _{\boldsymbol{v} \neq \mathbf{0}} \frac{\|\mathcal{C} v\|}{\|\boldsymbol{v}\|}=\sup _{\|\boldsymbol{v}\|=1}\|\mathcal{C} \boldsymbol{v}\| \leqslant 1
$$

A useful property of the operator norm used below is $\|\mathcal{C} v\| \leqslant\|\mathcal{C}\|\|\boldsymbol{v}\|$, which follows directly from its definition. Thus $\mathcal{C}$ is bounded and continuous, since, if $\lim _{n \rightarrow \infty} \boldsymbol{v}_{n}=\boldsymbol{v}$ then $\lim _{n \rightarrow \infty} \mathcal{C} v_{n}=\mathcal{C} v$ in $\mathcal{V}^{1}(V)$. Since $\mathcal{C}$ is bounded, it is the generator of a uniformly continuous semigroup (see Pazy 1983, § 1.1), $\mathcal{T}(t)=\exp (-t \mathcal{C})=\sum_{k=0}^{\infty}(-t \mathcal{C})^{k} / k !$. The solution of $\partial_{t} \boldsymbol{v}=-\mathcal{C} \boldsymbol{v}$ with $\boldsymbol{v}=\boldsymbol{v}_{0}$ at $t=0$ can be written in terms of the operator $\mathcal{T}$ as $\boldsymbol{v}=\mathcal{T}(t) \boldsymbol{v}_{0}$. 


\subsection{The spectrum of $\mathcal{C}$}

The spectrum of $\mathcal{C}$ may be defined in terms of its resolvent operator $(\mathcal{C}-\lambda I)^{-1}$ on $\mathcal{V}^{1}(V)$. The resolvent set $\Gamma$ of $\mathcal{C}$ is the set of $\lambda$ in the complex plane $\mathbb{C}$ for which $(\mathcal{C}-\lambda)^{-1}$ is a bounded operator. The forced solution of (2.6) is given in terms of the resolvent by $\boldsymbol{v}=(\mathcal{C}-\lambda \boldsymbol{I})^{-1} \boldsymbol{f}$. The solution is well behaved, and in particular no resonance occurs, if $\lambda$ lies in the resolvent set. The resolvent has been useful in the analysis of stability problems with non-normal operators (see the review by Trefethen 1997).

THEOREM 3.1. The resolvent set $\Gamma$ of $\mathcal{C}$ contains $\mathbb{C} \backslash[-\mathrm{i}, \mathrm{i}]$.

Proof. The inner product of (2.6) with $\boldsymbol{v}$ gives

$$
(\widehat{\boldsymbol{\Omega}} \times \boldsymbol{v}, \boldsymbol{v})-\lambda(\boldsymbol{v}, \boldsymbol{v})=(\boldsymbol{f}, \boldsymbol{v}),
$$

since $(\nabla p, v)=0$ by the divergence theorem, $\nabla \cdot v=0$ in $V$ and $\boldsymbol{n} \cdot \boldsymbol{v}=0$ on $\partial V$. The identity

$$
\left(\boldsymbol{v} \times \boldsymbol{v}^{*}\right)^{*}=-\boldsymbol{v} \times \boldsymbol{v}^{*},
$$

implies $\operatorname{Re}(\widehat{\boldsymbol{\Omega}} \times \boldsymbol{v}, \boldsymbol{v})=0$ and thus, if $\lambda_{r}, \lambda_{i}$ are the real and imaginary parts of $\lambda$,

$$
\lambda_{r}\|\boldsymbol{v}\|^{2}=-\operatorname{Re}(\boldsymbol{f}, \boldsymbol{v}), \quad \lambda_{i}\|\boldsymbol{v}\|^{2}=-\mathrm{i}(\widehat{\boldsymbol{\Omega}} \times \boldsymbol{v}, \boldsymbol{v})-\operatorname{Im}(\boldsymbol{f}, \boldsymbol{v}) .
$$

By the Cauchy-Schwartz inequality $|(\boldsymbol{f}, \boldsymbol{v})| \leqslant\|\boldsymbol{f}\|\|\boldsymbol{v}\|$ and $|(\widehat{\boldsymbol{\Omega}} \times \boldsymbol{v}, \boldsymbol{v})| \leqslant\|\boldsymbol{v}\|^{2}$. Hence

$$
\|\boldsymbol{v}\| \leqslant\left|\lambda_{r}\right|^{-1}\|\boldsymbol{f}\| ; \quad\|\boldsymbol{v}\| \leqslant\left(\left|\lambda_{i}\right|-1\right)^{-1}\|\boldsymbol{f}\|, \quad \text { if }\left|\lambda_{i}\right|>1 .
$$

Since $\boldsymbol{v}=(\mathcal{C}-\lambda \boldsymbol{I})^{-1} \boldsymbol{f},\left\|(\mathcal{C}-\lambda \boldsymbol{I})^{-1}\right\| \leqslant\left|\lambda_{r}\right|^{-1}$ and $\left\|(\mathcal{C}-\lambda \boldsymbol{I})^{-1}\right\| \leqslant\left(\left|\lambda_{i}\right|-1\right)^{-1}$ if $\left|\lambda_{i}\right|>1$. Hence the resolvent set contains all $\lambda$ such that $\lambda_{r} \neq 0$ or $\left|\lambda_{i}\right|>1$.

The spectrum of $\mathcal{C}$ is the complement $\mathbb{C} \backslash \Gamma$ of the resolvent set $\Gamma$ in the complex plane $\mathbb{C}$. Theorem 3.1 shows that the spectrum lies in $[-\mathrm{i}, \mathrm{i}]$ on the imaginary axis. In general, the spectrum is the disjoint union of the point (or discrete) spectrum $\sigma_{p}(\mathcal{C})$, the continuous spectrum $\sigma_{c}(\mathcal{C})$ and the residual spectrum $\sigma_{r}(\mathcal{C})$. The discrete spectrum $\sigma_{p}(\mathcal{C})$ consists of the eigenvalues of $\mathcal{C}$, i.e. the values of $\lambda \in \mathbb{C}$ for which $\mathcal{C} \boldsymbol{v}=\lambda \boldsymbol{v}$ for some non-zero $\boldsymbol{v} \in \mathcal{V}^{1}(V)$. These discrete modes, which we will refer to simply as Coriolis modes, are the free modes $(\boldsymbol{f}=\mathbf{0})$ of $\mathcal{C}$ and can be physically interpreted as inertial modes or geostrophic modes, or Rossby modes, of frequency $\omega=-\mathrm{i} \lambda$. As indicated in the introduction we regard the Coriolis modes of zero and non-zero frequency as the geostrophic modes and inertial spatial modes respectively. There is a further discussion of $\sigma_{c}(\mathcal{C})$ and $\sigma_{r}(\mathcal{C})$ in $\S 9$. The following theorem is important for the spectrum of $\mathcal{C}$, but particularly for $\sigma_{p}(\mathcal{C})$.

THEOREM 3.2. The operator $-\mathrm{i} \mathcal{C}$ is symmetric, i.e. $\left(-\mathrm{i} \mathcal{C} \boldsymbol{v}_{1}, \boldsymbol{v}_{2}\right)=\left(\boldsymbol{v}_{1},-\mathrm{i} \mathcal{C} \boldsymbol{v}_{2}\right)$ for all $\boldsymbol{v}_{1}, \boldsymbol{v}_{2} \in \mathcal{V}^{1}(V)$.

Proof. There exists a pressure $p_{1} \in \mathcal{P}$ such that $\left(-\mathrm{i} \mathcal{C} \boldsymbol{v}_{1}, \boldsymbol{v}_{2}\right)=\left(-\mathrm{i} \widehat{\boldsymbol{\Omega}} \times \boldsymbol{v}_{1}, \boldsymbol{v}_{2}\right)-$ $\mathrm{i}\left(\nabla p_{1}, \boldsymbol{v}_{2}\right)$. But $\left(\nabla p_{1}, \boldsymbol{v}_{2}\right)=0$, noting $\nabla \cdot \boldsymbol{v}_{2}=0$ in $V$, applying the divergence theorem and $\boldsymbol{n} \cdot \boldsymbol{v}_{2}=0$ on $\partial V$. Interchanging subscripts yields $\left(-i \mathcal{C} \boldsymbol{v}_{1}, \boldsymbol{v}_{2}\right)=\left(-\mathrm{C} \mathcal{C} \boldsymbol{v}_{2}, \boldsymbol{v}_{1}\right)^{*}=$ $\left(\boldsymbol{v}_{1},-\mathrm{i} \mathcal{C} \boldsymbol{v}_{2}\right)$, since $\mathrm{i} \widehat{\boldsymbol{\Omega}} \times \boldsymbol{v}_{1} \cdot \boldsymbol{v}_{2}^{*}=\left(\mathrm{i} \widehat{\boldsymbol{\Omega}} \times \boldsymbol{v}_{2} \cdot \boldsymbol{v}_{1}^{*}\right)^{*}$. 
The values $\lambda= \pm \mathrm{i}$ are not eigenvalues of $\mathcal{C}$, i.e. $\pm \mathrm{i} \notin \sigma_{p}(\mathcal{C})$. Physically, there are no Coriolis modes of frequency $\omega= \pm 1$, but if the fluid occupies all space $E^{3}$, modes with frequency $\omega= \pm 1$ are possible. Equation (2.6) with $\lambda=\mathrm{i}$ and $\boldsymbol{f}=\mathbf{0}$ implies $\widehat{\boldsymbol{\Omega}} \times \boldsymbol{v}=\mathrm{i} \boldsymbol{v}-\nabla p$ and hence $(\widehat{\boldsymbol{\Omega}} \times \boldsymbol{v}, \widehat{\boldsymbol{\Omega}} \times \boldsymbol{v})=(\mathrm{i} \boldsymbol{v}-\nabla p, \mathrm{i} \boldsymbol{v}-\nabla p)$. Simplifying using $(\boldsymbol{v}, \boldsymbol{\nabla} p)=(\boldsymbol{\nabla} p, \boldsymbol{v})^{*}=0$ yields $\|\nabla p\|^{2}+\|\widehat{\boldsymbol{\Omega}} \cdot \boldsymbol{v}\|^{2}=0$. Thus $p$ is constant and $\widehat{\boldsymbol{\Omega}} \cdot \boldsymbol{v}=0$. Equation (2.6) reduces to $\pm \mathrm{i} \boldsymbol{v}+\widehat{\boldsymbol{\Omega}} \times \boldsymbol{v}=\mathbf{0}$. From $\boldsymbol{\nabla} \cdot \boldsymbol{v}=0$, it follows that $\widehat{\boldsymbol{\Omega}} \cdot \boldsymbol{\nabla} \times \boldsymbol{v}=$ 0 . Hence, there exists a potential $\varphi(x, y, z)$ such that $v=\nabla_{\perp} \varphi$, where $\nabla_{\perp}$ is the gradient perpendicular to $\widehat{\boldsymbol{\Omega}}$ and $\nabla_{\perp}^{2} \varphi=0$. Multiplying the last equation by $\varphi^{*}$ and integrating by parts over a plane section of $V$ with $z$ constant yields $\int_{z=\text { const }}|\boldsymbol{v}|^{2} \mathrm{~d} A=0$, since $\boldsymbol{n} \cdot \boldsymbol{v}=0$ on the boundary $\partial V$. It follows that $\boldsymbol{v}=\mathbf{0}$ everywhere in $V$. The case $\lambda=-\mathrm{i}$ is similar.

\subsection{Eigenvalues and orthogonality of Coriolis modes}

Using the symmetry of $-\mathrm{i} \mathcal{C}$ from Theorem 3.2, we now show the eigenvalues of $\mathcal{C}$ are imaginary, i.e. the frequencies $\omega=-\mathrm{i} \lambda$ are real, and that Coriolis modes with different eigenvalues are orthogonal. We also use the boundedness of $\mathcal{C}$ to show $|\omega|=|\lambda| \leqslant 1$. These results are consistent with the restriction on the spectrum found in Theorem 3.1. We denote the pressure of the Coriolis modes by $P$.

THEOREM 3.3. The Coriolis modes have the following properties: the eigenvalues $\lambda$ of $\mathcal{C}$ are imaginary and $|\lambda| \leqslant 1$ : and modes $\left(\boldsymbol{v}_{1}, P_{1}, \lambda_{1}\right)$ and $\left(\boldsymbol{v}_{2}, P_{2}, \lambda_{2}\right)$ with different eigenvalues (frequencies) $\lambda_{1}=\mathrm{i} \omega_{1} \neq \lambda_{2}=\mathrm{i} \omega_{2}$ are orthogonal, $\left(\boldsymbol{v}_{1}, \boldsymbol{v}_{2}\right)=0$.

Proof. The proofs rely on the symmetry of $-\mathrm{i} \mathcal{C}$ and the boundedness of $\mathcal{C}$. Alternative proofs are given in Greenspan (1968). From $\omega=-\mathrm{i} \lambda,-\mathrm{i} \mathcal{C} v=\omega \boldsymbol{v}$. Since $-\mathrm{i} \mathcal{C}$ is symmetric and $\boldsymbol{v} \neq \mathbf{0}, \omega\|\boldsymbol{v}\|^{2}=(-\mathrm{i} \mathcal{C} v, \boldsymbol{v})=(\boldsymbol{v},-\mathrm{i} \mathcal{C} v)=\omega^{*}\|\boldsymbol{v}\|^{2}$, i.e. $\omega$ is real. Since $\|\mathcal{C}\| \leqslant 1,|\lambda|\|v\| \leqslant\|\lambda \boldsymbol{v}\|=\|\mathcal{C} v\| \leqslant\|\mathcal{C}\|\|v\| \leqslant\|v\|$, i.e. $|\lambda|<1$. Since $-\mathrm{i} \mathcal{C}$ is symmetric, $0=\left(-\mathrm{i} \mathcal{C} \boldsymbol{v}_{1}, \boldsymbol{v}_{2}\right)-\left(\boldsymbol{v}_{1},-\mathrm{i} \mathcal{C} \boldsymbol{v}_{2}\right)=\left(\omega_{1}-\omega_{2}\right)\left(\boldsymbol{v}_{1}, \boldsymbol{v}_{2}\right)$.

The orthogonality property of Theorem 3.3 does not apply to modes of the same eigenvalue. Thus, for any eigenvalue with an eigenspace of dimension greater than one, as is true for the geostrophic modes in a sphere, an orthogonal basis must be constructed, for example using the Gram-Schmidt method. For this purpose it is useful to express the inner product of two inertial modes $\left(\boldsymbol{v}_{1}, P_{1}, \lambda_{1}=\mathrm{i} \omega_{1}\right)$ and $\left(\boldsymbol{v}_{2}, P_{2}, \lambda_{2}=\right.$ $\mathrm{i} \omega_{2}$ ) in terms of the pressures $P_{1}$ and $P_{2}$. Equation (2.2) with $\boldsymbol{f}=\mathbf{0}$ or the dot product of (2.6) with $\widehat{\boldsymbol{\Omega}}$ if $\omega^{2} \neq 1$ gives the useful result

$$
\mathrm{i} \omega \widehat{\boldsymbol{\Omega}} \cdot \boldsymbol{v}=\widehat{\boldsymbol{\Omega}} \cdot \nabla P .
$$

Using (2.8) with $\boldsymbol{f}=\mathbf{0},(3.15)$, integrating over $V$ and using the boundary condition (1.6) yields, if $\omega_{1} \omega_{2} \neq 0$,

$$
\left(\boldsymbol{v}_{1}, \boldsymbol{v}_{2}\right)=\left(1-\omega_{1} \omega_{2}\right)^{-1} \int_{V}\left\{\nabla P_{1} \cdot \nabla P_{2}^{*}+\frac{1}{\omega_{1} \omega_{2}}\left(\widehat{\boldsymbol{\Omega}} \cdot \nabla P_{1}\right)\left(\widehat{\boldsymbol{\Omega}} \cdot \nabla P_{2}^{*}\right)\right\} \mathrm{d} V .
$$

Formula (3.16) fails if one or both modes are geostrophic, since then $\omega_{1} \omega_{2}=0$. The formula for the inner product of two geostrophic modes is derived in $\S 3.3$ below. 


\subsection{Inertial modes}

In some simple geometries inertial modes can be constructed analytically using the pressure. With $\boldsymbol{f}=\mathbf{0},(2.8)$ reduces to

$$
\mathrm{i} \omega\left(1-\omega^{2}\right) \boldsymbol{v}=-\omega^{2} \nabla P+(\widehat{\boldsymbol{\Omega}} \cdot \nabla P) \widehat{\boldsymbol{\Omega}}+\mathrm{i} \omega \widehat{\boldsymbol{\Omega}} \times \nabla P \text { in } V
$$

and

$$
-\omega^{2} \partial_{n} P+(\widehat{\boldsymbol{\Omega}} \cdot \nabla P) \boldsymbol{n} \cdot \widehat{\boldsymbol{\Omega}}+\mathrm{i} \omega \boldsymbol{n} \times \widehat{\boldsymbol{\Omega}} \cdot \nabla P=0 \quad \text { in } \partial V .
$$

Poincaré's equation (1.7) must be solved for $P$ subject to the boundary condition (3.18) and the velocity then determined from (3.17). The construction for a sphere (Bryan 1889) is outlined in $\S 4$ during the enumeration of the spherical inertial modes.

\subsection{Geostrophic modes}

The Coriolis modes of zero frequency, i.e. the geostrophic modes, require special treatment. Their properties can occasionally be found from those of inertial modes by taking the limit $\omega \rightarrow 0$. Thus the limit as $\omega \rightarrow 0$ of (3.15) yields $\widehat{\boldsymbol{\Omega}} \cdot \nabla P=0$, i.e. $P=P(\boldsymbol{r}-\boldsymbol{r} \cdot \widehat{\boldsymbol{\Omega}} \widehat{\boldsymbol{\Omega}})$, so these modes have no vertical structure.

The velocity $v$ can be found by substituting (3.15) into (3.17), dividing by i $\omega$ and then taking the limit $\omega \rightarrow 0$ to obtain $\boldsymbol{v}=\widehat{\boldsymbol{\Omega}} \times \nabla P+(\widehat{\boldsymbol{\Omega}} \cdot \boldsymbol{v}) \widehat{\boldsymbol{\Omega}}$, or by taking the cross product of (2.6) with $\widehat{\boldsymbol{\Omega}}$. The axial component $\widehat{\boldsymbol{\Omega}} \cdot \boldsymbol{v}$ of the flow is still undetermined. Letting $\omega_{1}, \omega_{2} \rightarrow 0$ in the inner product formula (3.16) using (3.15) leads to the undetermined axial components of $\boldsymbol{v}_{1}, \boldsymbol{v}_{2}$. We derive an expression for $\widehat{\boldsymbol{\Omega}} \cdot \boldsymbol{v}$ in terms of the pressure and the geometry following Greenspan (1968). Let $(x, y, z)$ be Cartesian coordinates with the $z$-axis aligned along $\widehat{\boldsymbol{\Omega}}$. We assume that any line parallel to $\widehat{\boldsymbol{\Omega}}$ intersects $\partial V$ in the top $z=z_{t}(x, y)$ and bottom $z=z_{b}(x, y)$ boundaries, with outward normals $\boldsymbol{n}_{t}:=\nabla\left(z-z_{t}\right)$ and $\boldsymbol{n}_{b}:=-\nabla\left(z-z_{b}\right)$. Clearly $\boldsymbol{n}_{t} \cdot \widehat{\boldsymbol{\Omega}}=1$ and $\boldsymbol{n}_{b} \cdot \widehat{\boldsymbol{\Omega}}=-1$. Imposing $\boldsymbol{n} \cdot \boldsymbol{v}=0$ on $\partial V$ yields $-\boldsymbol{n}_{t} \cdot \widehat{\boldsymbol{\Omega}} \times \nabla P=\widehat{\boldsymbol{\Omega}} \cdot \boldsymbol{v}=\boldsymbol{n}_{b} \cdot \widehat{\boldsymbol{\Omega}} \times \nabla P$ or $\nabla z_{t} \cdot \widehat{\boldsymbol{\Omega}} \times \nabla P=\nabla z_{b} \cdot \widehat{\boldsymbol{\Omega}} \times \nabla P$, i.e. $\nabla h \times \nabla z \cdot \nabla P=0$, where $h(x, y):=z_{t}-z_{b}$ is the height of the boundary. Hence, $P=P(h, z)$, if $\nabla h \neq \mathbf{0}$. But $\widehat{\boldsymbol{\Omega}} \cdot \nabla P=0$ implies $P=P(h)$ and so $\widehat{\boldsymbol{\Omega}} \cdot \boldsymbol{v}=\nabla z_{t} \cdot \widehat{\boldsymbol{\Omega}} \times \nabla P=\nabla z_{t} \times \nabla z_{b} \cdot \widehat{\boldsymbol{\Omega}} P^{\prime}(h)$. So the horizontal structure of geostrophic flows is restricted to one degree of freedom. Finally, using $\nabla z_{t} \times \nabla z_{b}=-\nabla\left(\left(z_{t}+z_{b}\right) / 2\right) \times \nabla h$,

$$
\boldsymbol{v}=\left[\widehat{\boldsymbol{\Omega}}-\nabla \frac{1}{2}\left(z_{t}+z_{b}\right)\right] \times \nabla P=P^{\prime}(h) \boldsymbol{t},
$$

where $\boldsymbol{t}:=\nabla\left[z-\left(z_{t}+z_{b}\right) / 2\right] \times \nabla h$ is tangent to the streamlines of $\boldsymbol{v}$. The inner product of two geostrophic modes with pressures $P_{1}$ and $P_{2}$ is

$$
\left(\boldsymbol{v}_{1}, \boldsymbol{v}_{2}\right)=\int_{V}\left[\widehat{\boldsymbol{\Omega}}-\nabla \frac{1}{2}\left(z_{t}+z_{b}\right)\right] \times \nabla P_{1} \cdot\left[\widehat{\boldsymbol{\Omega}}-\nabla \frac{1}{2}\left(z_{t}+z_{b}\right)\right] \times \nabla P_{2}^{*} \mathrm{~d} V .
$$

If $V$ is symmetrical about some $z$-plane, then $z_{t}+z_{b}$ is constant and the inner product reduces to

$$
\left(\boldsymbol{v}_{1}, \boldsymbol{v}_{2}\right)=\int_{V} \nabla P_{1} \cdot \nabla P_{2}^{*} \mathrm{~d} V
$$

An orthogonal set of geostrophic modes can be constructed by applying GramSchmidt orthogonalisation to a linearly independent set of geostrophic pressure modes using the inner product (3.21). 
The spectrum of $\mathcal{C}$ depends only on the geometry of $V$ and $\widehat{\boldsymbol{\Omega}}$, not on the rotation rate nor on any flow parameters. The simplest dependence is between closed curves and geostrophic modes. The intersection of the level surfaces of $z-\left(z_{t}+z_{b}\right) / 2$ and $h$, which depend only on $V$ and $\widehat{\boldsymbol{\Omega}}$, are called geostrophic curves, or contours if they lie on the boundary $z=z_{t}$ or $z=z_{b}$. The streamlines of the geostrophic flow (3.19) are geostrophic curves. If $G$ is a geostrophic curve,

$$
\oint_{G} \boldsymbol{v} \cdot \mathrm{d} \boldsymbol{l}=P^{\prime}(h) \oint_{G}|\boldsymbol{t}| \mathrm{d} l \neq 0 .
$$

There is no non-zero geostrophic flow along a curve which terminates on the boundary with $\boldsymbol{n} \cdot \boldsymbol{t} \neq 0$. If all curves are of this form there is no geostrophic flow, e.g. as in a sliced cylinder rotating about its axis, and therefore no Coriolis mode of frequency zero. Of course, curves need not terminate on the boundary, as in the case of a sliced cone rotating about its axis.

Geostrophic and inertial modes can be separated by frequency or orthogonality, but also geometrically by geostrophic cylinder average. The geostrophic contours that have the same boundary projections along $\widehat{\boldsymbol{\Omega}}$ generate a geostrophic cylinder of constant height, which need not be of circular cross-section. The geostrophic cylinder average of a vector field $\boldsymbol{F}$ over a geostrophic cylinder $C$ is

$$
\langle\boldsymbol{F}\rangle_{C}:=\frac{1}{|C|} \int_{C} \boldsymbol{F} \cdot \widehat{t} \mathrm{~d} S
$$

where $|C|$ is the surface area of $C$ and $\widehat{t}$ is the unit tangent field along the geostrophic curves generating $C$. We show that, if $C$ divides the fluid into two disjoint components $V=V_{1} \cup V_{2}$, then

$$
\omega\langle\boldsymbol{v}\rangle_{C}=0
$$

and hence for the inertial modes $(\omega \neq 0)$ the geostrophic cylinder average of $v$ vanishes: $\langle\boldsymbol{v}\rangle_{C}=0$. The proof follows from the geostrophic cylinder average of (2.6), $-\mathrm{i} \omega\langle\boldsymbol{v}\rangle_{C}+\langle\widehat{\boldsymbol{\Omega}} \times \boldsymbol{v}\rangle_{C}=-\langle\nabla p\rangle_{C}$ and

$$
0=\int_{V_{i}} \boldsymbol{\nabla} \cdot \boldsymbol{v} \mathrm{d} V=\int_{C} \boldsymbol{v} \cdot \mathrm{d} \boldsymbol{S}=\iint_{C} \boldsymbol{v} \cdot \mathrm{d} \boldsymbol{l} \times \widehat{\boldsymbol{\Omega}} \mathrm{d} z=|C|\langle\widehat{\boldsymbol{\Omega}} \times \boldsymbol{v}\rangle_{C},
$$

using $\boldsymbol{\nabla} \cdot \boldsymbol{v}=0$ in $V_{i}$ and $\boldsymbol{n} \cdot \boldsymbol{v}=0$ on the boundary. Also $\langle\nabla p\rangle_{C}=0$, since $\oint_{G} \boldsymbol{\nabla} p \cdot \mathrm{d} \boldsymbol{l}=$ 0 for any geostrophic contour $G$ on $C$. So, unlike geostrophic modes, inertial modes have vertical structure $\widehat{\boldsymbol{\Omega}} \cdot \nabla P \neq 0$ and hence an axial flow along the rotation axis by (3.15). The result (3.24) is also known as the mean circulation theorem (Greenspan 1968), that the circulation of a depth-averaged inertial (geostrophic) mode around a closed geostrophic contour is zero (non-zero).

\subsection{Angular momentum of Coriolis modes}

Inertial and geostrophic modes also differ in angular momentum effects. Geostrophic modes transmit the component of the pressure torque along the rotation axis between the ends of a geostrophic cylinder or annulus. Inertial modes lose torque to the angular momentum of the fluid. This mechanism of torque transfer is significant if the region is periphractic, i.e. the outer fluid boundary encloses one or more inner fluid boundaries so not every closed surface in $V$ can be continuously shrunk to a 
point without leaving $V$ (Maxwell 1891, pp. 18, 24, 25). For then the geostrophic modes transmit the axial component of the pressure torque from the outer boundary to the inner boundary (Hide 1995). The results follow from the identity

$$
\widehat{\boldsymbol{\Omega}} \cdot \int_{\partial C} \boldsymbol{r} \times P \mathrm{~d} \boldsymbol{S}=\mathrm{i} \omega \widehat{\boldsymbol{\Omega}} \cdot \boldsymbol{L}_{C}-\frac{\mathrm{d}\left(\widehat{\boldsymbol{\Omega}} \cdot \boldsymbol{I}_{C} \cdot \widehat{\boldsymbol{\Omega}}\right)}{\mathrm{d} t},
$$

where $\boldsymbol{I}_{C}$ and $\boldsymbol{L}_{C}$ are the inertia tensor of $C$ and the angular momentum of $C$ in the rotating frame, noting that $\widehat{\boldsymbol{\Omega}} \cdot \boldsymbol{I}_{C} \cdot \widehat{\boldsymbol{\Omega}}$ is constant if $C$ is a geostrophic cylinder or annulus. In dimensional form, $\boldsymbol{I}_{C}:=\int_{C} \rho\left(r^{2} \boldsymbol{I}-\boldsymbol{r r}\right) \mathrm{d} V$ and $\boldsymbol{L}_{C}:=\int_{C} \boldsymbol{r} \times \rho \boldsymbol{v} \mathrm{d} V$. Since $\widehat{\boldsymbol{\Omega}}$ is constant the last term in (3.26) vanishes, giving the result. For a sphere or spherical shell the result is trivial. Identity (3.26) is established by combining the dimensional identities

$$
\frac{\mathrm{d} \boldsymbol{I}_{C}}{\mathrm{~d} t}=\int_{C} \rho D_{t}\left(r^{2} \boldsymbol{I}-\boldsymbol{r r}\right) \mathrm{d} V+\int_{\partial C} \rho\left(r^{2} \boldsymbol{I}-\boldsymbol{r r}\right)\left(\boldsymbol{v}_{\partial C}-\boldsymbol{v}\right) \cdot \mathrm{d} \boldsymbol{S},
$$

$D_{t}\left(r^{2} \boldsymbol{I}-\boldsymbol{r r}\right)=2 \boldsymbol{r} \cdot \boldsymbol{v} \boldsymbol{I}-\boldsymbol{r} \boldsymbol{v}-\boldsymbol{v r}$ and $\boldsymbol{r} \times(\dot{\boldsymbol{\Omega}} \times \boldsymbol{r})=\left(r^{2} \boldsymbol{I}-\boldsymbol{r r}\right) \cdot \dot{\boldsymbol{\Omega}}$ to yield the following identity for the moments of the Coriolis and Poincaré forces,

$$
\begin{aligned}
\int_{C} \boldsymbol{r} & \times(\rho 2 \boldsymbol{\Omega} \times \boldsymbol{v}) \mathrm{d} V+\int_{C} \boldsymbol{r} \times(\rho \dot{\boldsymbol{\Omega}} \times \boldsymbol{r}) \mathrm{d} V \\
& +\int_{\partial C} \boldsymbol{\Omega} \cdot \rho\left(r^{2} \boldsymbol{I}-\boldsymbol{r} \boldsymbol{r}\right)\left(\boldsymbol{v}_{\partial C}-\boldsymbol{v}\right) \cdot \mathrm{d} \boldsymbol{S}=\frac{\mathrm{d}\left(\boldsymbol{\Omega} \cdot \boldsymbol{I}_{C}\right)}{\mathrm{d} t}+\boldsymbol{\Omega} \times \boldsymbol{L}_{C} .
\end{aligned}
$$

If $\dot{\boldsymbol{\Omega}}=\mathbf{0}$ and $C$ moves so that $\left(\boldsymbol{v}_{\partial C}-\boldsymbol{v}\right) \cdot \boldsymbol{n}=0$, which implies the angular momentum flux across the boundary $\partial C$ due to $\boldsymbol{\Omega}$ vanishes, the (dimensionless) identity reduces to

$$
\frac{\mathrm{d}\left(\widehat{\boldsymbol{\Omega}} \cdot \boldsymbol{I}_{C}\right)}{\mathrm{d} t}+\widehat{\boldsymbol{\Omega}} \times \boldsymbol{L}_{C}=\int_{C} \boldsymbol{r} \times(\widehat{\boldsymbol{\Omega}} \times \boldsymbol{v}) \mathrm{d} V=\mathrm{i} \omega \int_{C} \boldsymbol{r} \times \boldsymbol{v} \mathrm{d} V-\int_{\partial C} \boldsymbol{r} \times P \mathrm{~d} \boldsymbol{S} .
$$

The component along the rotation axis gives (3.26).

\subsection{Inversion of $\mathcal{C}-\lambda I$ and resonance}

Applications usually require, in principle, calculation of the resolvent $(\mathcal{C}-\lambda \boldsymbol{I})^{-1}$ and forced solutions $\boldsymbol{v}=(\mathcal{C}-\lambda \boldsymbol{I})^{-1} \boldsymbol{f}$, where $\boldsymbol{f}$ satisfies (1.16). For this the velocity can be eliminated as in (2.7) and (2.9), which can be solved for $p$. Equation (2.8) then gives $\boldsymbol{v}$. Alternatively, elimination of $p$ from (1.19) yields the vorticity equation,

$$
\partial_{t} \nabla \times \boldsymbol{v}-\widehat{\boldsymbol{\Omega}} \cdot \boldsymbol{\nabla} \boldsymbol{v}=\nabla \times \boldsymbol{f}
$$

or from (2.6) the modal form,

$$
\widehat{\boldsymbol{\Omega}} \cdot \nabla \boldsymbol{v}+\lambda \nabla \times \boldsymbol{v}=-\nabla \times \boldsymbol{f} .
$$

Uncurling recovers (1.19) or (2.6). In principle, if $\lambda$ is in the resolvent set $\Gamma$ of $\mathcal{C}$, this can be done for all $\boldsymbol{f} \in \mathcal{V}^{1}(V)$. However, if $\lambda$ is in the spectrum $\mathbb{C} \backslash \Gamma, \boldsymbol{f}$ may have to satisfy non-resonance conditions. 
We consider only the point spectrum $\lambda \in \sigma_{p}(\mathcal{C})$. If $\lambda=0$, (1.19) reduces to the steady form $\mathcal{C} v=f$ and the resolvent is then the inverse of $\mathcal{C}$. If a geostrophic mode exists, then $0 \in \sigma_{p}(\mathcal{C})$ and $\mathcal{C}$ is not invertible. Taylor (1963) considered this important case for the dynamo problem in the rapid-rotation limit $E, R o \rightarrow 0$. In this case the force $f$ is the (projected) Lorentz force $f_{L}$ and the force balance is magnetostrophic. We state a general existence theorem, Theorem 3.4, for solutions of $\mathcal{C} \boldsymbol{v}=\boldsymbol{f}$; a constructive proof is given in appendix A. We assume the region $V$ is axisymmetric with $\widehat{\boldsymbol{\Omega}}$ oriented along the symmetry $z$-axis. We assume the top and bottom boundaries, $z=z_{t}(s)$ and $z=z_{b}(s)$, where the cylindrical radius $s$ satisfies $s_{0} \leqslant s \leqslant s_{1}$, are smooth. For a given $\widehat{\boldsymbol{\Omega}}$, $V$ is geostrophically guided if there is exactly one geostrophic contour through each point on the boundary. We assume $V$ is geostrophically guided: $\nabla\left(z_{t}-z_{b}\right) \neq \mathbf{0}$ except at isolated values of $s$.

THEOREM 3.4. A necessary and sufficient condition for the existence of a singlevalued solution $\boldsymbol{v} \in \mathcal{V}^{1}(V)$ of

$$
\widehat{\boldsymbol{\Omega}} \cdot \nabla \boldsymbol{v}=-\nabla \times \boldsymbol{f}, \quad \nabla \cdot \boldsymbol{v}=0 \quad \text { in } V, \quad \boldsymbol{n} \cdot \boldsymbol{v}=g \quad \text { on } \partial V,
$$

where $f$ and $g$ are prescribed subject to the consistency condition $\int_{\partial V} g \mathrm{~d} S=0$, is

$$
|C(s)|\langle\boldsymbol{f}\rangle_{C(s)}+\int_{S(s)} g \mathrm{~d} S=0,
$$

for all geostrophic cylinders $C(s)$, where $S(s)$ are the caps of $C(s)$. The solution is only determined up to an arbitrary additive function of $s$ to $v_{\phi}$. If the further condition,

$$
\langle\boldsymbol{v}\rangle_{C(s)}=0 \text { for all } s_{0} \leqslant s \leqslant s_{1},
$$

is imposed, then the solution is unique.

The function $g$ allows for Ekman suction or a moving boundary. Imposing $g=0$ gives the result for the Coriolis operator.

COROLlary 3.1. A necessary and sufficient condition for the existence of a singlevalued solution $\boldsymbol{v} \in \mathcal{V}^{1}(V)$ of $\mathcal{C} \boldsymbol{v}=\boldsymbol{f}$ is that all geostrophic cylinder averages of $\boldsymbol{f}$ vanish,

$$
\langle\boldsymbol{f}\rangle_{C(s)}=0 \text {. }
$$

The non-resonance or solvability condition (3.35) is well known in dynamo theory as Taylor's (1963) condition for the magnetostrophic momentum equation. The theorem fails if $z_{t}(s)$ or $z_{b}(s)$ are not smooth, e.g. in a spherical shell. The proof produces a smooth solution $\boldsymbol{v}$ over each interval of $s$ for which $z_{t}(s)$ and $z_{b}(s)$ are smooth but the smooth pieces of $v$ do not fit together smoothly. This remains an outstanding problem (Hollerbach \& Proctor 1993; Hollerbach 1994a,b; Kleeorin et al. 1997; Dormy, Cardin \& Jault 1998; Soward \& Hollerbach 2000; Livermore \& Hollerbach 2012).

The invertibility of $\mathcal{C}$ can also be treated spectrally:

THEOREM 3.5. If $\boldsymbol{f}$ has an expansion in discrete Coriolis modes, $\boldsymbol{f}=\sum_{\alpha} f_{\alpha} \boldsymbol{v}_{\alpha}$ with $f_{\alpha}=\left(\boldsymbol{f}, \boldsymbol{v}_{\alpha}\right)$, then $\mathcal{C} \boldsymbol{v}=\boldsymbol{f}$ has the solution $\boldsymbol{v}=\sum_{\alpha} \mathrm{i} f_{\alpha} \boldsymbol{v}_{\alpha} / \omega_{\alpha}$, if the non-resonance or solvability conditions,

$$
\left(\boldsymbol{f}, \boldsymbol{v}_{\beta}\right)=0,
$$

hold for all $\omega_{\beta}=0$, i.e. for all geostrophic modes, and $\sum_{\alpha}\left|f_{\alpha} / \omega_{\alpha}\right|^{2}\left\|\boldsymbol{v}_{\alpha}\right\|^{2}<\infty$. 
The proof is elementary. The solvability conditions in Theorem 3.5 are equivalent to condition (3.34) in Theorem 3.4, but may be more tractable. If the Coriolis modes are complete, so that any $\boldsymbol{f}$ can be expanded in discrete Coriolis modes, then the spectral inversion in Theorem 3.5 works for any $\boldsymbol{f}$ which satisfies the solvability conditions. This highlights the usefulness and importance of completeness for the Coriolis modes, which we prove in $\S 8$ if $V$ is a sphere. For the general forced problem, $(\mathcal{C}-\lambda \boldsymbol{I})^{-1} \boldsymbol{f}=$ $\sum_{\alpha} \mathrm{i}\left(\omega-\omega_{\alpha}\right)^{-1} f_{\alpha} \boldsymbol{v}_{\alpha}$, where $\omega=-\mathrm{i} \lambda$, if $\left(\boldsymbol{f}, \boldsymbol{v}_{\beta}\right)=0$ for all $\beta$ for which $\omega=\omega_{\beta}$.

\section{Coriolis modes in a sphere}

In $\S \S 4-8$ the fluid region $V$ is the (non-dimensionalised) sphere $|\boldsymbol{r}|<1$. In the present section we enumerate the Coriolis modes in a sphere. The geostrophic modes require special treatment due to the zero frequency.

\subsection{Inertial pressure modes}

Poincaré's equation (2.7) in cylindrical polar coordinates $(s, \phi, z)$ with $\boldsymbol{f}=\mathbf{0}$ is

$$
s^{-1} \partial_{s}\left(s \partial_{s} P\right)+s^{-2} \partial_{\phi}^{2} P-\left(\omega^{-2}-1\right) \partial_{z}^{2} P=0, \quad s^{2}+z^{2}<1
$$

and the boundary condition $(2.8)$ is

$$
\omega^{2} s \partial_{s} P+\mathrm{i} \omega \partial_{\phi} P-\left(1-\omega^{2}\right) z \partial_{z} P=0, \quad s^{2}+z^{2}=1,
$$

since $\boldsymbol{n}=\boldsymbol{s} \mathbf{1}_{s}+z \mathbf{1}_{z}$. We transform the problem to the non-orthogonal frequencydependent spheroidal coordinates $(\xi, \zeta, \phi)$ (Bryan 1889) given by

$$
\sigma s=\sqrt{1-\zeta^{2}} \sqrt{1-\xi^{2}}, \quad \omega z=\xi \zeta, \quad 0 \leqslant \zeta \leqslant \omega, \omega \leqslant \xi \leqslant 1,
$$

where $\sigma^{2}+\omega^{2}=1$. The relation between $\sigma$ and $\omega$ removes the mixed second derivative from Poincaré's equation, allowing the variables to separate into Legendre's equation in $\zeta$ and in $\xi$, and also ensures the spherical boundary $s^{2}+z^{2}=1$ is a $\zeta$-surface, $\zeta=\omega$. The transformation is degenerate if $\omega=0$. The $\zeta$-surfaces ( $\xi$-surfaces) are nonconfocal oblate (prolate) spheroids with semi-axes $\sqrt{1-\zeta^{2}} / \sqrt{1-\omega^{2}}>1$ and $\zeta / \omega<1$ (respectively, $\sqrt{1-\xi^{2}} / \sqrt{1-\omega^{2}}<1$ and $\xi / \omega>1$ ), co-axial with the $z$-axis. Thus the spherical inertial modes are

$$
P=P_{n, \omega}^{m}:=P_{n}^{m}\left(\zeta_{\omega}\right) P_{n}^{m}\left(\xi_{\omega}\right) \mathrm{e}^{\mathrm{i} m \phi},
$$

where $P_{n}^{m}$ is the associated Legendre function of degree $n$ and order $m, m \in \mathbb{Z},|m| \leqslant n$, $\omega \neq 0$ and the $\omega$-dependence of $\zeta$ and $\xi$ is indicated.

The product $P_{n}^{m}(\xi) P_{n}^{m}(\zeta)$ is a polynomial in $s$ and $z$ of degree $n$, and $P_{n}^{m}(\xi) P_{n}^{m}(\zeta) \mathrm{e}^{\mathrm{i} m \phi}$ is a polynomial in $x, y, z$ of degree $n$ (Kudlick 1966; Zhang et al. 2001), even if $\sigma^{2}+$ $\omega^{2} \neq 1$. In fact,

$$
P_{n, \omega}^{m}= \begin{cases}\left(\sigma s \mathrm{e}^{\mathrm{i} \phi}\right)^{m}(\omega z)^{q} U_{n}^{m}\left(\sigma^{2} s^{2}, \omega^{2} z^{2}\right), & \text { if } m \geqslant 0 \\ \left(\sigma s \mathrm{e}^{-\mathrm{i} \phi}\right)^{|m|}(\omega z)^{q} U_{n}^{m}\left(\sigma^{2} s^{2}, \omega^{2} z^{2}\right), & \text { if } m<0\end{cases}
$$

where $q:=n-|m|(\bmod 2)$ is either 0 or 1 , and $U_{n}^{m}\left(\sigma^{2} s^{2}, \omega^{2} z^{2}\right)$ is a polynomial in $\sigma^{2} s^{2}$ and $\omega^{2} z^{2}$ of degree $(n-|m|-q) / 2$. To establish this, note the associated Legendre function $P_{n}^{m}(w)=\left(1-w^{2}\right)^{|m| / 2} w^{q} u_{n}^{m}\left(w^{2}\right)$, where $u_{n}^{m}\left(w^{2}\right)$ is a polynomial 
in $w^{2}$ of degree $(n-|m|-q) / 2$. Thus $P_{n, \omega}^{m}=\left(\sigma s \mathrm{e}^{\mathrm{i} \phi}\right)^{m}(\omega z)^{q} u_{n}^{m}\left(\xi^{2}\right) u_{n}^{m}\left(\zeta^{2}\right)$ if $m \geqslant 0$; and $P_{n, \omega}^{m}=\left(\sigma s \mathrm{e}^{-\mathrm{i} \phi}\right)^{|m|}(\omega z)^{q} u_{n}^{m}\left(\xi^{2}\right) u_{n}^{m}\left(\zeta^{2}\right)$ if $m<0$. The result (4.5) follows, since the product $u_{n}^{m}\left(\xi^{2}\right) u_{n}^{m}\left(\zeta^{2}\right)$ is a polynomial $U_{n}^{m}\left(\sigma^{2} s^{2}, \omega^{2} z^{2}\right)$ in $(\sigma s)^{2}$ and $(\omega z)^{2}$ of degree $(n-|m|-q) / 2$ with coefficients independent of $\omega$ and $\sigma$ by the following lemma. Expressions (4.5) are polynomials in $x, y, z$ since $s \mathrm{e}^{ \pm \mathrm{i} \phi}=x \pm \mathrm{i} y$. The $z$-parity of $P_{n, \omega}^{m}$, which is not immediately apparent from $\zeta$ and $\xi$, is determined by $q$.

LEMMA 4.1. Let $s_{1}=\sqrt{1-\zeta^{2}} \sqrt{1-\xi^{2}}, z_{1}=\xi \zeta$. If $p(w)$ is a polynomial of degree $K$, then $p\left(\xi^{2}\right) p\left(\zeta^{2}\right)$ is a polynomial in $s_{1}^{2}$ and $z_{1}^{2}$ of degree $K$.

Proof. Let $p(w)=\sum_{k=0}^{K} p_{k} w^{k}$, where the $p_{k}$ are constants. Then

$$
p\left(\xi^{2}\right) p\left(\zeta^{2}\right)=\sum_{k=0}^{K}\left\{p_{k}^{2} z_{1}^{2 k}+\sum_{l=1}^{K-k} p_{k} p_{k+l} z_{1}^{2 k}\left(\xi^{2 l}+\zeta^{2 l}\right)\right\} .
$$

By rearranging the binomial expansion of $\left(\xi^{2}+\zeta^{2}\right)^{l}$ in the form,

$$
\xi^{2 l}+\zeta^{2 l}=\left(\xi^{2}+\zeta^{2}\right)^{l}-\sum_{j=1}^{\left\lfloor\frac{l-1}{2}\right\rfloor}{ }^{l} C_{j}(\xi \zeta)^{2 j}\left(\xi^{2(l-2 j)}+\zeta^{2(l-2 j)}\right)
$$

and using the identity $\xi^{2}+\zeta^{2}=1-s_{1}^{2}+z_{1}^{2}$, it follows by induction that $\xi^{2 l}+\zeta^{2 l}$ is a polynomial in $s_{1}^{2}$ and $z_{1}^{2}$ of degree $l$. Hence $p\left(\xi^{2}\right) p\left(\zeta^{2}\right)$ is a polynomial in $s_{1}^{2}$ and $z_{1}^{2}$ of degree $K$.

The boundary $s^{2}+z^{2}=1$ is given by $s=\sin \theta$ and $z=\cos \theta$, or in oblate spheroidal coordinates by $\xi=\cos \theta$ and $\zeta=\omega$. The boundary condition implies $\omega$ is a root of

$$
\left(1-\omega^{2}\right) \frac{\mathrm{d} P_{n}^{m}(\omega)}{\mathrm{d} \omega}+m P_{n}^{m}(\omega)=0 .
$$

Now $P_{n}^{m}(\omega)=\left(1-\omega^{2}\right)^{|m| / 2} p_{n}^{m}(\omega)$, where $p_{n}^{m}(\omega)$ is a polynomial in $\omega$ of degree $n-|m|$. Moreover, $\left\{p_{n}^{m}(\omega)\right\}_{n=|m|}^{\infty}$ form an orthogonal set of polynomials on $[-1,1]$ with weight function $\left(1-\omega^{2}\right)^{|m|}$ even in $\omega$, since

$$
\frac{1}{2} \int_{-1}^{1} P_{n}^{m}(\omega) P_{N}^{m}(\omega) \mathrm{d} \omega=\delta_{n N} .
$$

Thus $p_{n}^{m}(\omega)$ has $n-|m|$ simple zeros $\omega_{k}, k=1: n-|m|$, on $(-1,1)$ symmetric about $\omega=0$ and hence $p_{n}^{m}(\omega)=c_{n}^{m} \prod_{k=1}^{n-|m|}\left(\omega-\omega_{k}\right)$, where $c_{n}^{m}$ is a constant. The frequency equation (3.4) can be written as $\mathrm{d} \ln P_{n}^{m}(\omega) / \mathrm{d} \omega=-m /\left(1-\omega^{2}\right)$ or

$$
\sum_{k=1}^{n-|m|} \frac{1}{\omega-\omega_{k}}=-\frac{\frac{1}{2}(|m|+m)}{\omega+1}-\frac{\frac{1}{2}(|m|-m)}{\omega-1} .
$$

For $m \neq 0$ this equation has $n-|m|$ zeros; for $m=0$ there are $n-1$ zeros. Figure 1 shows the generic case for $n-|m|=4$. The zeros are not symmetrically distributed about $\omega=0$. Geostrophic modes $\omega=0$ occur if and only if $m=0$ and $n$ is even. There are no $m= \pm n$ modes: in fact, the dispersion relation gives $\omega=1(-1)$ for 


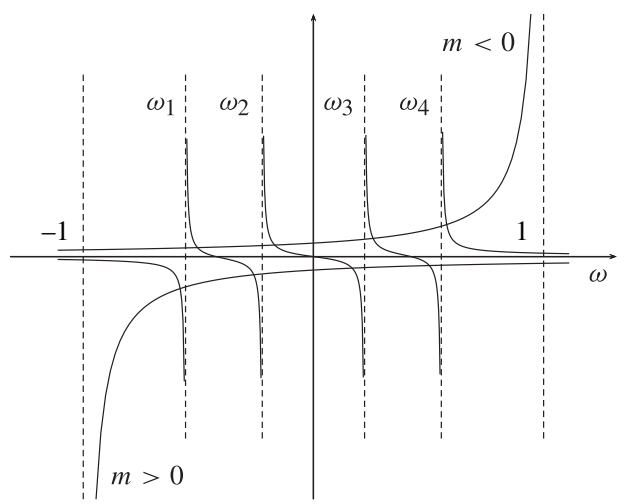

FIGURE 1. Schematic plot of the two sides of (5.6) for inertial frequencies $\omega$ with $n-$ $|m|=4$. The right-hand side curves are labelled by $m>0$ and $m<0$; the $m=0$ curve is the $\omega$ axis.

$m>0(<0)$ in these cases. Thus we label the non-zero frequencies for $0<m \leqslant n-1$ by $\omega_{n, j}^{m}$, where $j \in \mathbb{Z}, j \neq 0$ and $j=-(n-m+1) / 2:(n-m-1) / 2$, if $n-m$ is odd, or $j=-(n-m) / 2:(n-m) / 2$, if $n-m$ is even. The zero frequencies are $\omega_{n, 0}^{0}$, where $n$ is even, $m=0$ and $j=0$. For $-(n-1) \leqslant m<0, \omega_{n, j}^{m}=\omega_{n,-j}^{-m}$. We define the associated inertial pressure mode for the non-zero frequency $\omega_{n, j}^{m}$ by

$$
P_{n, j}^{m}:=P_{n, \omega_{n, j}^{m}}^{m}
$$

Note that $P_{n, j}^{m}=P_{n,-j}^{-m *}$. We construct the geostrophic modes in $\S 4.2$.

The number of Coriolis pressure modes of degree $N+1$ or less, which equals the number of Coriolis velocity modes of degree $N$ or less, is

$$
C_{N}=\sum_{n=1}^{N+1} \sum_{m=-n}^{n}\left(n-|m|-\delta_{0}^{m}\right)=\frac{1}{6} N(N+1)(2 N+7) .
$$

For $N=1,2,3$ there are 3,11, 26 modes and for $N \gg 1 C_{N} \approx N^{3} / 3$. Greenspan (1968) counts $n-|m|(n-|m|-1)$ inertial modes if $n-m$ is even (odd), in agreement with (4.12).

\subsection{Geostrophic pressure modes}

We construct the geostrophic modes by taking the limit $\omega \rightarrow 0$ in the solutions (4.4). They are the natural choice, since they are hierarchical in polynomial degree. These geostrophic modes can also be constructed by applying Gram-Schmidt orthogonalisation with the inner product (1.11) to the polynomials $\left\{1, s^{2}, s^{4}, \ldots\right\}$. See figure 2. Other geostrophic bases are possible.

LEMMA 4.2. For $m=0$ and $\omega \rightarrow 0$ the solutions (4.4) reduce to the geostrophic modes,

$$
P_{n, 0}^{0}(s)=P_{n}(0) P_{n}\left(\sqrt{1-s^{2}}\right)-\left[P_{n}(0)\right]^{2},
$$

where $n$ is even. Here, $P_{n, 0}^{0}(s)$ is a polynomial in $s^{2}$ of even degree $n$ in $s$ and $P_{n}$ is the Legendre polynomial of degree $n$. 


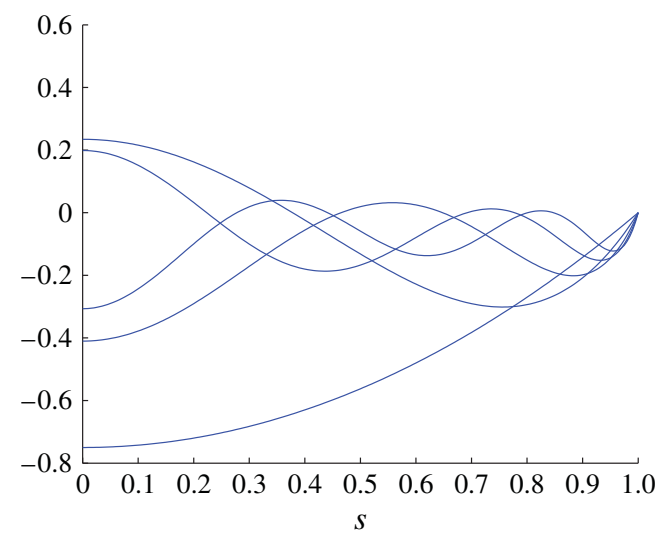

FIGURE 2. (Colour online) The geostrophic modes $P_{n, 0}^{0}$ as a function of cylindrical radius $s$ for $n=2,4,6,8,10$.

Proof. From (4.3) $\xi^{2}+\zeta^{2}=1-\sigma^{2} s^{2}+(\xi \zeta)^{2}=1-\sigma^{2} s^{2}+\omega^{2} z^{2}$. Thus as $\omega \rightarrow 0$, $\xi^{2}+\zeta^{2} \rightarrow 1-s^{2}$ and, by (4.7), $\xi^{2 l}+\zeta^{2 l} \rightarrow\left(\xi^{2}+\zeta^{2}\right)^{l}$, since $\sigma \rightarrow 1$ and $(\xi \zeta)^{2}=$ $\omega^{2} z^{2} \rightarrow 0$. If $n$ is even, $P_{n}(z)=\sum_{k=0}^{n / 2} p_{n, 2 k} z^{2 k}$, where the $p_{n, 2 k}$ are constants, and

$$
P_{n}(\xi) P_{n}(\zeta)=p_{n, 0}\left[\sum_{k=0}^{n / 2} p_{n, 2 k}\left(\xi^{2 k}+\zeta^{2 k}\right)-p_{n, 0}\right]+\sum_{k=1}^{n / 2} \sum_{l=1}^{n / 2} p_{n, 2 k} p_{l, 2 k} \xi^{2 k} \zeta^{2 l}
$$

The double sum is $O(\xi \zeta)^{2}$. Thus, for $n$ even, $P_{n}(\xi) P_{n}(\zeta) \rightarrow P_{n, 0}^{0}$ in (4.13) as $\omega \rightarrow 0$, since $p_{n, 0}=P_{n}(0)$. If $n$ is odd, $P_{n}(\xi) P_{n}(\zeta) \rightarrow 0$ as $\omega \rightarrow 0$.

Linearly independent Coriolis modes of the same frequency may be non-orthogonal. The modes (4.13) are orthogonal: evaluating the inner product $\left(\boldsymbol{v}_{1}, \boldsymbol{v}_{2}\right)$ of two modes using (3.21), noting $n_{1}$ and $n_{2}$ are even and substituting $v^{2}=1-s^{2}$ yields, apart from the factor $P_{n_{1}}(0) P_{n_{2}}(0)$,

$$
\int_{V} \nabla P_{n_{1}}\left(\sqrt{1-s^{2}}\right) \cdot \nabla P_{n_{2}}\left(\sqrt{1-s^{2}}\right) \mathrm{d} V=2 \pi \int_{-1}^{1} P_{n_{1}}^{\prime}(v)\left(1-v^{2}\right) P_{n_{2}}^{\prime}(v) \mathrm{d} v .
$$

An integration by parts and Legendre's differential equation shows the last integral vanishes if $n_{1} \neq n_{2}$.

\subsection{Velocity modes}

The velocity modes are constructed from the pressure modes using (3.17). Thus associated with the pressure mode $P_{n+1, j}^{m}$ is the velocity mode $\boldsymbol{v}_{n, j}^{m}$. Define

$$
\boldsymbol{v}_{n}^{m}(\omega):=\left\{\begin{array}{l}
\frac{\mathrm{i} \omega}{1-\omega^{2}}\left(\nabla P_{n+1, \omega}^{m}-\omega^{-2} \widehat{\boldsymbol{\Omega}} \widehat{\boldsymbol{\Omega}} \cdot \nabla P_{n+1, \omega}^{m}\right)+\frac{1}{1-\omega^{2}} \widehat{\boldsymbol{\Omega}} \times \nabla P_{n+1, \omega}^{m}, \quad \omega \neq 0 \\
\widehat{\boldsymbol{\Omega}} \times \nabla P_{n+1,0}^{0}, \quad \omega=0, m=0, n \text { odd } .
\end{array}\right.
$$

The Coriolis velocity modes are thus

$$
\boldsymbol{v}_{n, j}^{m}:=N_{n, j}^{m} \boldsymbol{v}_{n}^{m}\left(\omega_{n+1, j}^{m}\right), \quad|m| \leqslant n,
$$


where $j=-(n-m+1) / 2:(n-m-1) / 2$ if $n-m$ is odd, $j=-(n-m) / 2:(n-m) / 2$ if $n-m$ is even, and $j \neq 0$ unless $m=0$ and $n$ is odd. The normalisation constant $N_{n, j}^{m}>0$ is chosen so that the mode is normalised to $\left(\boldsymbol{v}_{n, j}^{m}, \boldsymbol{v}_{n, j}^{m}\right)=1$. The complex conjugate $\boldsymbol{v}_{n, j}^{m *}=\boldsymbol{v}_{n,-j}^{-m}$. No modes $\boldsymbol{v}_{n, \omega}^{m}$ arise from $m=n+1$, i.e. $P_{n+1}^{ \pm(n+1)}$. For $m \neq 0$ the associated inertial waves propagate eastward (westward) if $j>0(j<0)$. Equatorial symmetries are determined by $n-m$ : the velocity modes are quadrupole symmetric if $n-m=$ $0(\bmod 2)$ and dipole symmetric if $n-m=1(\bmod 2)$; and rotational symmetries about $\widehat{\boldsymbol{\Omega}}$ are determined by $m$. The velocities of the geostrophic modes (4.13) agree with Liao \& Zhang (2010a) up to normalisation and phase: in terms of Jacobi polynomials their $\phi$-components can be written as

$$
v_{2 n-1,0}^{0}=N_{2 n-1,0}^{0} \frac{\mathrm{d} P_{2 n, 0}^{0}(s)}{\mathrm{d} s}=N_{2 n-1,0}^{0}(-)^{n}(2 n+1) P_{2 n}(0) s P_{n}^{(1 / 2,1)}\left(2 s^{2}-1\right) .
$$

Below we label the Coriolis modes using a 3-index Greek letter, e.g. $\boldsymbol{v}_{\alpha} \equiv \boldsymbol{v}_{n_{\alpha}, j_{\alpha}}^{m_{\alpha}}$, and denote the degree of the polynomial flow $\boldsymbol{v}_{\alpha}$ by $\operatorname{deg} \alpha$.

\section{Polynomial flows in a sphere}

Let $\mathcal{P}_{N}$ be the space of polynomials in $(x, y, z)$ of degree $N$ or less with complex coefficients. The space $\mathcal{P}_{N}^{3}$ of polynomial vectors in $(x, y, z)$ of degree $N$ or less with complex coefficients has finite dimension

$$
\operatorname{dim} \mathcal{P}_{N}^{3}=3 \sum_{0 \leqslant i+j+k \leqslant N} 1=\frac{1}{2}(N+1)(N+2)(N+3) .
$$

We define the space of (inviscid) complex polynomial flows of degree $N$ on $V$, $\mathcal{P}_{N}(V)=\left\{\boldsymbol{v} \in \mathcal{P}_{N}^{3} \mid \nabla \cdot \boldsymbol{v}=0\right.$ in $V, \boldsymbol{n} \cdot \boldsymbol{v}=0$ on $\left.\partial V\right\}$ with inner product (1.11). It is not immediately obvious, due to the boundary condition (1.5), that $\mathcal{P}_{N}(V)$ contains non-zero flows; for a sphere $\boldsymbol{n}$ is a polynomial. We now show that, if $V$ is a sphere, the dimension of $\mathcal{P}_{N}(V)$ is $C_{N}$ given by (4.12), the number of Coriolis modes of degree $N$ or less. Since $\mathcal{P}_{N}(V) \subsetneq \mathcal{P}_{N}^{3}, \operatorname{dim} \mathcal{P}_{N}(V)<\frac{1}{2}(N+1)(N+2)(N+3)$.

Constructing or even enumerating a basis of $\mathcal{P}_{N}(V)$ from linear combinations of the power basis $\left\{x^{i} y^{j} z^{k} \boldsymbol{i}, x^{i} y^{j} z^{k} \boldsymbol{j}, x^{i} y^{j} z^{k} \boldsymbol{k}\right\}, i, j, k \in \mathbb{N}$ and $i+j+k \leqslant N$, of $\mathcal{P}_{N}^{3}$ is difficult due to the solenoidal condition and particularly the boundary condition (1.5). It is much simpler to construct a basis for $\mathcal{P}_{N}(V)$ using spherical toroidal and poloidal fields: if $\boldsymbol{v} \in \mathcal{P}_{N}(V)$ then $\boldsymbol{v}=\boldsymbol{S}\{s\}+\boldsymbol{T}\{t\}$, where

$$
\boldsymbol{T}\{t\}:=\nabla \times \operatorname{tr}, \quad \boldsymbol{S}\{s\}:=\nabla \times \boldsymbol{T}\{s\}, \quad \oint t \mathrm{~d} \Omega=0, \quad \oint s \mathrm{~d} \Omega=0,
$$

with $t \in \mathcal{P}_{N}, s \in \mathcal{P}_{N+1}$ and $\mathrm{d} \Omega=\sin \theta \mathrm{d} \theta \mathrm{d} \phi$ the element of solid angle, and conversely if $t \in \mathcal{P}_{N}, s \in \mathcal{P}_{N+1}$ then $\boldsymbol{v} \in \mathcal{P}_{N}(V)$. Note that $t$ and $s$ are not the time nor the cylindrical radius in this section. The boundary condition (1.5) is equivalent to $s=0$ on $r=1$. Indeed, the Cartesian component of $\boldsymbol{v}$ in the direction of a constant vector $\boldsymbol{c}$ is $\boldsymbol{c} \cdot \boldsymbol{v}=$ $-(\boldsymbol{c} \cdot \boldsymbol{r}) \nabla^{2} s+\boldsymbol{c} \cdot \nabla(\boldsymbol{r} \cdot \nabla s)-\boldsymbol{c} \times \boldsymbol{r} \cdot \nabla t$. Thus, if $t \in \mathcal{P}_{N}$ and $s \in \mathcal{P}_{N+1}$, then $\boldsymbol{v} \in \mathcal{P}_{N}^{3}$. Conversely, if $v \in \mathcal{P}_{N}^{3}$, then $t \in \mathcal{P}_{N}$ and $s \in \mathcal{P}_{N+1}$, since for any spherical harmonic $Y_{n}^{m}$ of degree $n \geqslant 1$ and order $m, \Lambda^{-2} f(r) Y_{n}^{m}=-f(r) Y_{n}^{m} /[n(n+1)]$ for any radial function $f(r)$, where $\boldsymbol{\Lambda}:=\boldsymbol{r} \times \nabla$. 
To construct a polynomial toroidal-poloidal basis of $\mathcal{P}_{N}(V)$ and for the theorems in $\S 6$, we need the spherical harmonic expansion of a polynomial $f \in \mathcal{P}_{N}$ (see MacRobert 1947, p. 140),

$$
f=\sum_{n=0}^{N} \sum_{p=n}^{N} \sum_{m=-n}^{n} f_{n, p}^{m} r^{p} Y_{n}^{m}(\theta, \phi)
$$

where the $f_{n, p}^{m}$ are constant and the prime on the $p$-sum indicates summation over $p=$ $n, n+2, n+4, \ldots$ The polynomial $f$ is not necessarily harmonic. Thus, noting (5.2), the potentials $t \in \mathcal{P}_{N}$ and $s \in \mathcal{P}_{N+1}$ have spherical harmonic expansions of the form

$$
\begin{gathered}
t=\sum_{n=1}^{N} \sum_{m=-n}^{n}\left(\sum_{j=0}^{\left\lfloor\frac{1}{2}(N-n)\right\rfloor} t_{n, j}^{m} r^{2 j}\right) r^{n} Y_{n}^{m}(\theta, \phi), \\
s=\sum_{n=1}^{N+1} \sum_{m=-n}^{n}\left(\sum_{j=0}^{\left\lfloor\frac{1}{2}(N+1-n)\right\rfloor} \tilde{s}_{n, j}^{m} r^{2 j}\right) r^{n} Y_{n}^{m}(\theta, \phi),
\end{gathered}
$$

where the coefficients $\tilde{s}_{n, j}^{m}$ are adjusted below to satisfy the boundary condition $s=0$ on $r=1$. The dimension of the toroidal subspace $\mathcal{P}_{N}^{\mathrm{T}}$ of $\mathcal{P}_{N}(V)$ is

$$
\operatorname{dim} \mathcal{P}_{N}^{\mathrm{T}}=\sum_{n=1}^{N} \sum_{m=-n}^{n} \sum_{j=0}^{\left\lfloor\frac{1}{2}(N-n)\right\rfloor} 1= \begin{cases}\frac{1}{6} N\left(N^{2}+6 N+8\right), & N \text { even; } \\ \frac{1}{6}(N+1)\left(N^{2}+5 N+3\right), & N \text { odd }\end{cases}
$$

The boundary condition (1.5) must still be imposed on the poloidal flows: on $r=1$, $\sum_{j=0}^{\left\lfloor\frac{1}{2}(N+1-n)\right\rfloor} \tilde{s}_{n, j}^{m}=0$. Elimination of $\tilde{s}_{n, 0}^{m}$ gives

$$
s=\sum_{n=1}^{N-1} \sum_{m=-n}^{n}\left(\sum_{j=1}^{\left\lfloor\frac{1}{2}(N+1-n)\right\rfloor} \tilde{s}_{n, j}^{m}\left(r^{2 j}-1\right)\right) r^{n} Y_{n}^{m}(\theta, \phi),
$$

from which it is clear that $1-r^{2}$ is a factor of $s$. Hence we can write

$$
s=\left(1-r^{2}\right) \sum_{n=1}^{N-1} \sum_{m=-n}^{n}\left(\sum_{j=0}^{\left\lfloor\frac{1}{2}(N+1-n)\right\rfloor-1} s_{n, j}^{m} r^{2 j}\right) r^{n} Y_{n}^{m}(\theta, \phi),
$$

where the new coefficients $s_{n, j}^{m}$ are independent. Thus the dimension of the poloidal subspace $\mathcal{P}_{N}^{\mathrm{P}}$ of $\mathcal{P}_{N}(V)$ is

$$
\operatorname{dim} \mathcal{P}_{N}^{\mathrm{P}}=\sum_{n=1}^{N-1} \sum_{m=-n}^{n} \sum_{j=0}^{\left\lfloor\frac{1}{2}(N+1-n)\right\rfloor-1} 1= \begin{cases}\frac{1}{6} N\left(N^{2}+3 N-1\right), & N \text { even } \\ \frac{1}{6}(N-1)(N+1)(N+3), & N \text { odd }\end{cases}
$$

Finally,

$$
\operatorname{dim} \mathcal{P}_{N}(V)=\operatorname{dim} \mathcal{P}_{N}^{\mathrm{T}}(V)+\operatorname{dim} \mathcal{P}_{N}^{\mathrm{P}}(V)=\frac{1}{6} N(N+1)(2 N+7)=C_{N},
$$

i.e. the dimension of $\mathcal{P}_{N}(V)$ equals the number of Coriolis velocity modes of degree $N$ or less as in (4.12). If $\boldsymbol{t}_{n, j}^{m}:=\nabla \times\left\{r^{n+2 j} Y_{n}^{m}(\theta, \phi) \boldsymbol{r}\right\}$, then $\left\{\boldsymbol{t}_{n, j}^{m}\right\}$, where $n=1: N$, $|m| \leqslant n, j=0:\left\lfloor\frac{1}{2}(N-n)\right\rfloor$, is a basis of $\mathcal{P}_{N}^{\mathrm{T}}(V)$. Similarly, if $\boldsymbol{s}_{n, j}^{m}:=\nabla \times \nabla \times\{(1-$ $\left.\left.r^{2}\right) r^{n+2 j} Y_{n}^{m}(\theta, \phi) \boldsymbol{r}\right\}$, then $\left\{\boldsymbol{s}_{n, j}^{m}\right\}$ for $n=1: N-1,|m| \leqslant n, j=0:\left\lfloor\frac{1}{2}(N+1-n)\right\rfloor-1$ is a basis of $\mathcal{P}_{N}^{\mathrm{P}}(V)$. 


\section{The Coriolis operator on polynomial flows in a sphere}

If the Coriolis operator $\mathcal{C}$ is restricted to act on polynomial flows $\mathcal{P}_{N}(V)$ of degree $N$ or less, it is not true that $\mathcal{C}: \mathcal{P}_{N}(V) \rightarrow \mathcal{P}_{N}(V)$ for any $V$, i.e. that it produces only polynomial flows of degree $N$ or less. However, if $V$ is a sphere the restricted Coriolis operator $\mathcal{C}: \mathcal{P}_{N}(V) \rightarrow \mathcal{P}_{N}(V)$ is well defined, i.e. $\mathcal{C}$ maps polynomial flows to polynomial flows of the same degree. This is the essential result of the paper, Theorem 6.3, and follows from the fundamental tool, Theorem 6.2.

THEOREM 6.1 (Polynomial Laplace-Neumann Problem in a Sphere). There exists a unique solution $\varphi$ of Laplace's equation $\nabla^{2} \varphi=0$ in the sphere $V(r<a)$, subject to $\boldsymbol{n} \cdot \nabla \varphi=q$ on $\partial V$, where $\boldsymbol{n}(=\boldsymbol{r} / a)$ is a linear polynomial and $q \in \mathcal{P}_{N_{1}}$, and to the respective uniqueness and consistency conditions,

$$
\int_{V} \varphi \mathrm{d} V=0, \quad \int_{\partial V} q \mathrm{~d} S=0,
$$

and $\varphi \in \mathcal{P}_{N_{1}}$.

Proof. Since $\boldsymbol{n}=\boldsymbol{r} / a, \boldsymbol{n} \cdot \nabla \varphi=q$ reduces to $\partial_{r} \varphi=q$ on $r=a$. On $r=a, \partial_{r} \varphi=$ $\sum_{n=1}^{N_{1}} \sum_{m=-n}^{n} q_{n}^{m} Y_{n}^{m}(\theta, \phi)$, since $q \in \mathcal{P}_{N_{1}}$. The general solution of $\nabla^{2} \varphi=0$ is $\varphi=$ $\sum_{n=1}^{\infty} \sum_{m=-n}^{n} \varphi_{n}^{m}(r / a)^{n} Y_{n}^{m}(\theta, \phi)$. Thus $\varphi_{n}^{m}=q_{n}^{m} / n$ for $1 \leqslant n \leqslant N_{1}$ and $\varphi_{n}^{m}=0$ for $n>N_{1}$, i.e. $\varphi \in \mathcal{P}_{N_{1}}$.

LEMmA 6.1 (Polynomial Integral of the Polynomial Poisson-Neumann Problem in $E^{3}$ ). If $f \in \mathcal{P}_{N_{2}}$, then there exists a solution $\varphi_{p}$ of Poisson's equation $\nabla^{2} \varphi=f$ with $\varphi_{p} \in \mathcal{P}_{N_{2}+2}$ in $E^{3}$.

Proof. Since $f \in \mathcal{P}_{\mathrm{N}_{2}}$, it can be expanded in spherical harmonics as in (5.3). Define

$$
\varphi_{p}:=\sum_{n=0}^{N_{2}} \sum_{p=n}^{N_{2}} \sum_{m=-n}^{n} \varphi_{n, p}^{m} r^{p+2} Y_{n}^{m}(\theta, \phi),
$$

where $\varphi_{n, p}^{m}=f_{n, p}^{m}[(p+2)(p+3)-n(n+1)]^{-1}$ and $p \geqslant n$. Then $\varphi_{p} \in \mathcal{P}_{N_{2}+2}$ and $\nabla^{2} \varphi_{p}=$ $f$.

The fundamental theorem of the paper is

Theorem 6.2 (Polynomial Poisson-Neumann Problem in a Sphere). There exists a unique solution $\varphi$ of Poisson's equation $\nabla^{2} \varphi=f$ in the sphere $V(r<a)$ subject to $f \in \mathcal{P}_{N_{2}}, \boldsymbol{n} \cdot \nabla \varphi=q$ on $\partial V$, where $\boldsymbol{n}=\boldsymbol{r} / a$ and $q \in \mathcal{P}_{N_{1}}$, and to the respective uniqueness and consistency conditions

$$
\int_{V} \varphi \mathrm{d} V=0, \quad \int_{V} f \mathrm{~d} V=\int_{\partial V} q \mathrm{~d} S,
$$

and $\varphi \in \mathcal{P}_{N_{3}}$, where $N_{3}=\max \left(N_{1}, N_{2}+2\right)$. 
Proof. By Lemma 6.1 there exists a polynomial $\varphi_{p} \in \mathcal{P}_{N_{2}+2}$ such that $\nabla^{2} \varphi_{p}=f$ and $\int_{V} \varphi_{p} \mathrm{~d} V=0$. Thus $\nabla^{2}\left(\varphi-\varphi_{p}\right)=0$ in $V$ and $\boldsymbol{n} \cdot \nabla\left(\varphi-\varphi_{p}\right)=q-\boldsymbol{n} \cdot \nabla \varphi_{p}$ on $\partial V$. Since the uniqueness and consistency conditions of Theorem 6.1 hold, i.e. $\int_{V}\left(\varphi-\varphi_{p}\right) \mathrm{d} V=0$ and

$$
\int_{\partial V}\left(q-\boldsymbol{n} \cdot \nabla \varphi_{p}\right) \mathrm{d} S=\int_{\partial V} q \mathrm{~d} S-\int_{V} \nabla^{2} \varphi_{p} \mathrm{~d} V=\int_{\partial V} q \mathrm{~d} S-\int_{V} f \mathrm{~d} V=0,
$$

Theorem 6.1 applies and implies $\varphi-\varphi_{p} \in \mathcal{P}_{N_{1}}$. Hence $\varphi \in \mathcal{P}_{N_{3}}$.

The essential theorem of the paper is that $\mathcal{C}$ maps polynomial flows of degree $N$ or less in a sphere to polynomial flows of degree $N$ or less.

THEOREM 6.3 (Invariance of Polynomial Flows in a Sphere). The space $\mathcal{P}_{N}(V)$ of polynomial flows of degree $N$ or less on a sphere $V$ is invariant under $\mathcal{C}$, i.e. $\mathcal{C} \mathcal{P}_{N}(V) \subseteq \mathcal{P}_{N}(V)$.

Proof. To show the Coriolis operator $\mathcal{C}$ is well defined on $\mathcal{P}_{N}(V)$ we must show for $\boldsymbol{v} \in \mathcal{P}_{N}(V)$ that the pressure $p$ exists, $p \in \mathcal{P}_{N+1}$ and $\mathcal{C} \boldsymbol{v}=\widehat{\boldsymbol{\Omega}} \times \boldsymbol{v}+\nabla p \in \mathcal{P}_{N}(V)$. Note that $\boldsymbol{v}$ is a polynomial flow, but not necessarily an inertial mode, i.e. of the form (4.4). We can construct $p$ as in (1.10) so that $\nabla \cdot(\mathcal{C} v)=\widehat{\boldsymbol{\Omega}} \cdot \nabla \times \boldsymbol{v}+\nabla^{2} p=0$ in $V$ and $\boldsymbol{n} \cdot(\mathcal{C} \boldsymbol{v})=\boldsymbol{n} \cdot \widehat{\boldsymbol{\Omega}} \times \boldsymbol{v}+\boldsymbol{n} \cdot \nabla p=0$ on $\partial V$, by Theorem 6.2 with $f=-\widehat{\boldsymbol{\Omega}} \cdot \boldsymbol{\nabla} \times \boldsymbol{v} \in \mathcal{P}_{N-1}$ and $q=-\boldsymbol{n} \cdot \widehat{\boldsymbol{\Omega}} \times \boldsymbol{v} \in \mathcal{P}_{N+1}$. Thus $N_{1}=N+1$ and $N_{2}=N-1$ in that theorem, i.e. $N_{3}=N+1$ and hence $p \in \mathcal{P}_{N+1}$. Of course, $\nabla p \in \mathcal{P}_{N}^{3}$ and thus $\mathcal{C} \boldsymbol{v} \in \mathcal{P}_{N}(V)$.

We now show the operator $\mathcal{C}$ on $\mathcal{P}_{N}(V)$ is non-defective, i.e. its eigenvectors span $\mathcal{P}_{N}(V)$. In fact, $-\mathrm{i} \mathcal{C}$ can be represented by a hermitian matrix. The proof is simple on the finite-dimensional space $\mathcal{P}_{N}(V)$ and does not require the explicit construction of the modes as a basis of $\mathcal{P}_{N}(V)$. The result is used to prove the completeness of the Coriolis modes in the infinite-dimensional space $\mathcal{V}^{1}(V)$ in Theorem 8.1.

Theorem 6.4 (Existence and Polynomial Nature of Coriolis Modes in a Sphere). The finite-dimensional operator $-\mathrm{i} \mathcal{C}$ on $\mathcal{P}_{N}(V)$ is self-adjoint. The eigenvalues $\lambda=\mathrm{i} \omega$ of $\mathcal{C}$ are purely imaginary and satisfy $|\lambda|=|\omega|<1$ and the eigenvectors of distinct eigenvalues of $\mathcal{C}$ are orthogonal. The eigenvectors of $\mathcal{C}$ span the finite-dimensional space $\mathcal{P}_{N}(V)$. The number of Coriolis modes of degree $N$ or less is $C_{N}$.

Proof. If $\boldsymbol{v}_{1}, \boldsymbol{v}_{2} \in \mathcal{P}_{N}(V)$, then $\left(-\mathrm{i} \mathcal{C} \boldsymbol{v}_{1}, \boldsymbol{v}_{2}\right)=\left(\boldsymbol{v}_{1},-\mathrm{i} \mathcal{C} \boldsymbol{v}_{2}\right)$ by Theorem 3.2 and $-\mathrm{i} \mathcal{C}$ is self-adjoint since $\mathcal{P}_{N}(V)$ is finite-dimensional. Thus the eigenvectors of $-\mathrm{i} \mathcal{C}$ span $\mathcal{P}_{N}(V)$, the eigenvectors of distinct eigenvalues are orthogonal and its eigenvalues are real. If $\boldsymbol{v}$ is an eigenvector of $-\mathrm{i} \mathcal{C}$ corresponding to the eigenvalue $\omega$, then $-\mathrm{i} \mathcal{C} v=\omega \boldsymbol{v}$ and hence $\mathcal{C} \boldsymbol{v}=\lambda \boldsymbol{v}$, i.e. $\boldsymbol{v}$ is an eigenvector of $\mathcal{C}$ corresponding to the eigenvalue $\lambda=\mathrm{i} \omega$. Thus the eigenvectors of $\mathcal{C}$ are complete in the finite-dimensional space $\mathcal{P}_{N}(V)$ and its eigenvalues are purely imaginary. The bound $|\omega|<1$ follows as in Theorem 3.3 and the result that $\omega \neq \pm 1$.

The spaces $\mathcal{P}_{N}(V)$ are hierarchical in the sense that if $N_{1} \leqslant N_{2}$ and $(\boldsymbol{v}, \omega)$ is an eigenmode of $\mathcal{C}$ in $\mathcal{P}_{N_{1}}(V)$ then $(\boldsymbol{v}, \omega)$ is an eigenmode of $\mathcal{C}$ in $\mathcal{P}_{N_{2}}(V)$. Moreover, $(\boldsymbol{v}, \omega)$ is an eigenmode of $\mathcal{C}$ in $\mathcal{V}^{1}(V)$. Moreover, for any polynomial flow $\boldsymbol{v} \in \mathcal{P}_{N}(V)$, $\left(\boldsymbol{v}, \boldsymbol{v}_{\alpha}\right)=0$ for all Coriolis modes $\boldsymbol{v}_{\alpha}$ with $\operatorname{deg} \alpha>N$. 


\section{The Stokes and other operators in the Coriolis basis}

The Coriolis operator $\mathcal{C}$ restricted to polynomial flows in a sphere $V$ of degree $n$ or less $\mathcal{P}_{n}(V)$ is a diagonal $C_{n} \times C_{n}$ matrix in the Coriolis basis $\boldsymbol{v}_{\alpha}$ with $\operatorname{deg} \alpha \leqslant n$. What forms do other operators restricted to $\mathcal{P}_{n}(V)$ take in the Coriolis basis? Consider the operator $\nabla^{2}$ : if $\boldsymbol{v} \in \mathcal{P}_{n}(V)$, it does not follow that $\nabla^{2} \boldsymbol{v} \in \mathcal{P}_{n}(V)$, due to the impenetrable boundary condition. Instead, we can adjust $\nabla^{2} \boldsymbol{v}$ by subtracting the gradient of a harmonic function $p$, where $p$ is the solution of the Poisson-Neumann problem (1.14). This leads naturally to the Stokes operator $\mathcal{S} v:=-\nabla^{2} \boldsymbol{v}+\nabla p$. Since $\boldsymbol{n} \cdot \nabla^{2} \boldsymbol{v} \in \mathcal{P}_{n-1}$, it follows from Theorem 6.2 that $p \in \mathcal{P}_{n-1}$ and $\mathcal{S} \boldsymbol{v} \in \mathcal{P}_{n-2}(V)$. Hence, for any $\boldsymbol{v} \in \mathcal{P}_{n}(V)$ there exist coefficients $u_{\alpha}$ such that

$$
\mathcal{S} v=\sum_{\operatorname{deg} \alpha \leqslant n-2} u_{\alpha} \boldsymbol{v}_{\alpha}
$$

and for any $\boldsymbol{v}_{\beta}$ with $\operatorname{deg} \beta \geqslant n-1$,

$$
\left(\nabla^{2} \boldsymbol{v}, \boldsymbol{v}_{\beta}\right)=\left(\nabla^{2} \boldsymbol{v}, \boldsymbol{v}_{\beta}\right)-\left(\nabla p, \boldsymbol{v}_{\beta}\right)=-\left(\mathcal{S} \boldsymbol{v}, \boldsymbol{v}_{\beta}\right)=-\sum_{\operatorname{deg} \alpha \leqslant n-2} u_{\alpha}\left(\boldsymbol{v}_{\alpha}, \boldsymbol{v}_{\beta}\right)=0,
$$

since $\left(\nabla p, \boldsymbol{v}_{\beta}\right)=0$. In particular, $I_{n, j, N, J}^{m, m}=0$ if $n \leqslant N+1$, which is consistent with Liao \& Zhang (2009). The Stokes matrix in the Coriolis basis decomposes further on subspaces of $\mathcal{P}_{N}(V)$ invariant under rotations about the rotation axis and subspaces invariant under reflection in the equator. Analogous results hold for the operators $\partial_{x}^{2}$, $\partial_{y}^{2}, \partial_{z}^{2}, s^{-1} \partial_{s}\left(s \partial_{s}\right)$ or $s^{-2} \partial_{\phi}^{2}$.

The advection operator $\mathcal{A}\left(\boldsymbol{v}_{1}, \boldsymbol{v}_{2}\right):=\boldsymbol{v}_{1} \cdot \nabla \boldsymbol{v}_{2}+\nabla p$ maps two polynomial flows $\boldsymbol{v}_{i} \in$ $\mathcal{P}_{n_{i}}(V), \quad i=1,2$, of degrees $n_{1}$ and $n_{2}$ to a polynomial flow of degree $n_{1}+n_{2}-1$, if $p$ satisfies (1.17) with $\boldsymbol{F}=\mathcal{A}\left(\boldsymbol{v}_{1}, \boldsymbol{v}_{2}\right)$. In particular, for any three Coriolis modes $\boldsymbol{v}_{\alpha}$, $\boldsymbol{v}_{\beta}$ and $\boldsymbol{v}_{\gamma}$, if $\operatorname{deg} \alpha+\operatorname{deg} \beta \leqslant \operatorname{deg} \gamma$ or $m_{\alpha}+m_{\beta} \neq m_{\gamma}$,

$$
\left(\boldsymbol{v}_{\alpha} \cdot \nabla \boldsymbol{v}_{\beta}, \boldsymbol{v}_{\gamma}\right)=\int_{V}\left(\boldsymbol{v}_{\alpha} \cdot \nabla \boldsymbol{v}_{\beta}\right) \cdot \boldsymbol{v}_{\gamma}^{*} \mathrm{~d} V=0 .
$$

Thus the operator $\mathcal{A}$ maps $\mathcal{P}_{N}(V) \times \mathcal{P}_{N}(V)$ to $\mathcal{P}_{2 N-1}(V)$. In fact, if $\boldsymbol{v} \in \mathcal{P}_{N}(V)$, there exist coefficients $u_{\alpha}$ such that

$$
\mathcal{A}(\boldsymbol{v}, \boldsymbol{v})=\sum_{\operatorname{deg} \alpha \leqslant 2 N-1} u_{\alpha} \boldsymbol{v}_{\alpha}
$$

and for any $\boldsymbol{v}_{\beta}$ with $\operatorname{deg} \beta \geqslant 2 N,\left(\boldsymbol{v} \cdot \nabla \boldsymbol{v}, \boldsymbol{v}_{\beta}\right)=\left(\mathcal{A}(\boldsymbol{v}, \boldsymbol{v}), \boldsymbol{v}_{\beta}\right)=0$.

\section{Completeness of the Coriolis modes in a sphere}

In this section we establish in Theorem 8.1 the most important result in the paper, that the Coriolis modes in a sphere are complete in $\mathcal{V}^{1}(V)$. The proof relies on the uniform polynomial approximation theorem of Weierstrass to approximate a flow in $\mathcal{V}^{1}(V)$ by a polynomial vector field and the fundamental Theorem 6.2 to project this polynomial field onto $\mathcal{P}_{N}(V)$ for sufficiently high degree $N$ by subtracting a pressure gradient.

THEOREM 8.1. The Coriolis modes in a sphere $V$ are complete in $\mathcal{V}^{1}(V)$. 
Proof. Let $v \in \mathcal{V}^{1}(V)$ and $\epsilon>0$. By application of the Weierstrass approximation theorem (see Davis 1975, §6.6) to each Cartesian component of $\boldsymbol{v}$, there exists an integer $N>0$ and a vector polynomial $\boldsymbol{u} \in \mathcal{P}_{N}^{3}$, such that $|\boldsymbol{v}-\boldsymbol{u}|<\epsilon$ in $V$. However, $\boldsymbol{u} \in \mathcal{P}_{N}$ may be false, since the conditions $\boldsymbol{\nabla} \cdot \boldsymbol{u}=0$ in $V$ and $\boldsymbol{n} \cdot \boldsymbol{u}=0$ on $\partial V$ do not necessarily hold. By Theorem 6.2 there exists a polynomial $\pi \in \mathcal{P}_{N+1}$ such that $\boldsymbol{u}-\nabla \pi \in \mathcal{P}_{N}$. Hence there are constants $u_{\alpha}$ such that

$$
\boldsymbol{u}=\nabla \pi+\sum_{\operatorname{deg} \alpha \leqslant N} u_{\alpha} \boldsymbol{v}_{\alpha}=\nabla \pi+\sum_{\operatorname{deg} \alpha \leqslant N}\left(\boldsymbol{u}, \boldsymbol{v}_{\alpha}\right) \boldsymbol{v}_{\alpha}
$$

where the $\boldsymbol{v}_{\alpha}$ are the orthonormal Coriolis modes in $\mathcal{P}_{N}(V)$. Now, using (8.1),

$$
\begin{aligned}
\| & \boldsymbol{v} \\
= & \sum_{\operatorname{deg} \alpha \leqslant N}\left(\boldsymbol{v}, \boldsymbol{v}_{\alpha}\right) \boldsymbol{v}_{\alpha}\left\|^{2}=\right\|(\boldsymbol{v}-\boldsymbol{u}+\nabla \pi)+\sum_{\operatorname{deg} \alpha \leqslant N}\left(\boldsymbol{u}-\boldsymbol{v}, \boldsymbol{v}_{\alpha}\right) \boldsymbol{v}_{\alpha} \|^{2} \\
= & \|\boldsymbol{v}-\boldsymbol{u}+\nabla \pi\|^{2}+\sum_{\operatorname{deg} \alpha \leqslant N}\left(\boldsymbol{u}-\boldsymbol{v}, \boldsymbol{v}_{\alpha}\right)^{*}\left(\boldsymbol{v}-\boldsymbol{u}+\nabla \pi, \boldsymbol{v}_{\alpha}\right) \\
& +\sum_{\operatorname{deg} \alpha \leqslant N}\left(\boldsymbol{u}-\boldsymbol{v}, \boldsymbol{v}_{\alpha}\right)\left(\boldsymbol{v}_{\alpha}, \boldsymbol{v}-\boldsymbol{u}+\nabla \pi\right)+\left\|\sum_{\operatorname{deg} \alpha \leqslant N}\left(\boldsymbol{u}-\boldsymbol{v}, \boldsymbol{v}_{\alpha}\right) \boldsymbol{v}_{\alpha}\right\|^{2} \|^{2} \\
= & (\boldsymbol{v}-\boldsymbol{u}+\nabla \pi, \boldsymbol{v}-\boldsymbol{u})-\sum_{\operatorname{deg} \alpha \leqslant N}\left|\left(\boldsymbol{u}-\boldsymbol{v}, \boldsymbol{v}_{\alpha}\right)\right|^{2} \\
\leqslant & \|\boldsymbol{v}-\boldsymbol{u}\|^{2}+\|\nabla \pi\|\|\boldsymbol{v}-\boldsymbol{u}\|,
\end{aligned}
$$

since $(\boldsymbol{v}, \nabla \pi)=0,(\boldsymbol{u}-\nabla \pi, \nabla \pi)=0$ as $\boldsymbol{u}-\nabla \pi \in \mathcal{P}_{N},\left(\nabla \pi, \boldsymbol{v}_{\alpha}\right)=0$ and $\left(\boldsymbol{v}_{\alpha}, \boldsymbol{v}_{\beta}\right)=$ $\delta_{\alpha \beta}$. We bound $\|\nabla \pi\|$ in terms of $\|\boldsymbol{v}\|$. Now $(\boldsymbol{u}-\nabla \pi, \nabla \pi)=0$ implies $\|\nabla \pi\|^{2}=$ $(\boldsymbol{u}, \nabla \pi) \leqslant\|\boldsymbol{u}\|\|\nabla \pi\|$, i.e. $\|\nabla \pi\| \leqslant\|\boldsymbol{u}\|$, and $|\boldsymbol{u}-\boldsymbol{v}| \leqslant \epsilon$ implies $\|\boldsymbol{u}-\boldsymbol{v}\| \leqslant \epsilon \sqrt{|V|}$. Thus $\|\nabla \pi\| \leqslant\|\boldsymbol{u}\|=\|\boldsymbol{v}+(\boldsymbol{u}-\boldsymbol{v})\| \leqslant\|\boldsymbol{v}\|+\|\boldsymbol{u}-\boldsymbol{v}\| \leqslant\|\boldsymbol{v}\|+\epsilon \sqrt{|V|}$, where $|V|$ is the volume of $V$; i.e. $\|\nabla \pi\| \leqslant\|v\|+\epsilon \sqrt{|V|}$. Thus finally,

$$
\left\|\boldsymbol{v}-\sum_{\operatorname{deg} \alpha \leqslant N}\left(\boldsymbol{v}, \boldsymbol{v}_{\alpha}\right) \boldsymbol{v}_{\alpha}\right\|^{2} \leqslant \sqrt{|V|}(\|\boldsymbol{v}\|+2 \epsilon \sqrt{|V|}) \epsilon .
$$

Since the right-hand side is independent of $N, \boldsymbol{u}$ and $\pi$, it follows that $\boldsymbol{v}=$ $\sum_{\alpha}\left(\boldsymbol{v}, \boldsymbol{v}_{\alpha}\right) \boldsymbol{v}_{\alpha}$ in $\mathcal{V}^{1}(V)$, where the sum is over Coriolis modes. The Coriolis modes are therefore complete in a sphere.

We showed that the Coriolis modes are polynomials by construction in $\S 4$. We now show this also follows from Theorem 6.2 and that there are no missed eigenfunctions.

THEOREM 8.2. The spherical Coriolis modes are polynomials.

Proof. Let $\boldsymbol{v}=\sum_{\alpha} v_{\alpha} \boldsymbol{v}_{\alpha}$ and $\mathcal{C} \boldsymbol{v}=\sum_{\alpha} \lambda_{\alpha} v_{\alpha} \boldsymbol{v}_{\alpha}$, then $\|\boldsymbol{v}\|^{2}=\sum_{\alpha}\left|v_{\alpha}\right|^{2}$ and $\|\mathcal{C} \boldsymbol{v}\|^{2}=$ $\sum_{\alpha}\left|\lambda_{\alpha}\right|^{2}\left|v_{\alpha}\right|^{2}$. If $(\boldsymbol{v}, \lambda)$ is an eigenmode, then $0=\|(\mathcal{C}-\lambda \boldsymbol{I}) \boldsymbol{v}\|^{2}=\sum_{\alpha}\left|\lambda_{\alpha}-\lambda\right|^{2}\left|v_{\alpha}\right|^{2}$. The series all converge since the kinetic energy is finite, $\|\boldsymbol{v}\|<\infty$, and $\left|\lambda_{\alpha}\right|<1$ for all $\alpha$. Thus $\lambda=\lambda_{\beta}$ for some index $\beta, v_{\beta} \neq 0$ and $v_{\alpha}=0$ for $\alpha \neq \beta$. 
The operator $\mathcal{C}$ can be approximated elementwise by a finite-rank operator $\mathcal{C}_{N}$, i.e. an operator with a finite-dimensional range. Let $\boldsymbol{P}_{N}$ be the projection onto $\mathcal{P}_{N}$, $\boldsymbol{v}_{N}=\boldsymbol{P}_{N} \boldsymbol{v}:=\sum_{\operatorname{deg} \alpha \leqslant N}\left(\boldsymbol{v}, \boldsymbol{v}_{\alpha}\right) \boldsymbol{v}_{\alpha}$ and $\mathcal{C}_{N}:=\mathcal{C} \circ \boldsymbol{P}_{N}: \mathcal{V}^{1}(V) \rightarrow \mathcal{P}_{N}(V)$. Then

$$
\left\|\left(\mathcal{C}-\mathcal{C}_{N}\right) \boldsymbol{v}\right\|=\left\|\mathcal{C}\left(\boldsymbol{v}-\boldsymbol{v}_{N}\right)\right\| \leqslant\|\mathcal{C}\|\left\|\boldsymbol{v}-\boldsymbol{v}_{N}\right\| \leqslant \sqrt{\sqrt{|V|}(1+2 \epsilon \sqrt{|V|})} \sqrt{\epsilon}
$$

using (8.3). However, the approximation is non-uniform, since $N$ in (8.3) depends not only on $\epsilon$ but also $\boldsymbol{v}$. Hence it does not follow from (8.4) that for all $\epsilon>0$ there exists $N$ such that $\mathcal{C}_{N}$ approximates $\mathcal{C}$ in the uniform operator norm, i.e. $\left\|\mathcal{C}-\mathcal{C}_{N}\right\|=$ $\sup _{\|\boldsymbol{v}\|=1}\left\|\left(\mathcal{C}-\mathcal{C}_{N}\right) \boldsymbol{v}\right\|<\epsilon$, which would imply that $\mathcal{C}$ is a compact operator. In fact, we show in the next theorem that $\mathcal{C}$ is not compact and hence cannot be uniformly approximated by the $\mathcal{C}_{N}$ or any finite-rank operator (Schechter 1971, §4.3).

THEOREM 8.3. The Coriolis operator $\mathcal{C}$ is not compact if $V$ is a sphere.

Proof. We prove the theorem by contradiction. Assume $\mathcal{C}$ is compact. Then the only point of accumulation of the frequencies $\omega_{n, j}^{m}$ of $\mathcal{C}$ is zero (Friedman 1982, Corollary 5.3.3), i.e. for all $\delta>0$ there are finitely many frequencies $\omega$ with $\delta<|\omega|<1$. However, from $\S 4$ the $m=0$ frequencies $\omega_{n, j}^{0}$ are interlaced by the zeros $\omega_{j}$ of $P_{n}$ which satisfy $\cos [2 j \pi /(2 n+1)] \leqslant \omega_{j} \leqslant \cos [(2 j-1) \pi /(2 n+1)]$, i.e. there exists a $\delta>0$ such that $\delta<|\omega|<1$ contains infinitely many $m=0$ frequencies, a contradiction. Hence $\mathcal{C}$ is not compact.

Despite the lack of a uniform finite-dimensional approximation of $\mathcal{C}$, Theorem 8.3 importantly precludes an accumulation of near-resonance conditions, i.e. $\left(\boldsymbol{f}, \boldsymbol{v}_{\beta}\right)=$ $O\left(\omega_{\beta}\right)\|\boldsymbol{f}\|$ with $\left|\omega_{\beta}\right| \ll 1$ compared to (3.36). Although Theorem 8.3 is only a partial result, the proof shows that $\min _{n \leqslant N, j \neq 0}\left|\omega_{n, j}^{0}\right|=O\left(N^{-1}\right)$, which indicates that with axisymmetry frequencies near zero behave uniformly with $N$ and allow some regularity in inverting $\mathcal{C}$. The behaviour is also true for $m \neq 0$, since $\min _{n \leqslant N, m, j \neq 0}\left|\omega_{n, j}^{m}\right|$ is $O\left(N^{-1}\right)$, using the estimate $\omega_{n, j}^{|m|}=-\sin \{\pi j /(n+(1 / 2))\}+o\left(n^{-1}\right)$ (see Ivanov \& Papaloizou 2010), equation (B5) with $j$ shifted so that $\omega_{n, 0}^{|m|}=0$. Thus the convergence condition on $\boldsymbol{v}$ in Theorem 8.3 becomes $\sum_{\alpha}\left|f_{\alpha} \operatorname{deg} \alpha\right|^{2}<$ $\infty$, where the sum is over the spherical inertial modes. Splitting the sum into $O\left[\delta(\operatorname{deg} \alpha)^{2}\right]$ frequencies in the range $O(\operatorname{deg} \alpha)^{-1}<\left|\omega_{\alpha}\right| \leqslant \delta<1$ near 0 and $O\left[(1-\delta)(\operatorname{deg} \alpha)^{2}\right]$ frequencies in $\delta<\left|\omega_{\alpha}\right|<1$ gives an upper bound of order $\sum_{n=1}^{\infty}\left[n^{4} \delta+n^{2} \delta^{-2}(1-\delta)\right] \max _{\operatorname{deg} \alpha=n}\left|f_{\alpha}\right|^{2}$. Minimising over $\delta$ yields $\delta=O(\operatorname{deg} \alpha)^{-2 / 3}$ and the convergence condition $\sum_{n=1}^{\infty}\left(n^{5 / 3} \max _{\operatorname{deg} \alpha=n}\left|f_{\alpha}\right|\right)^{2}<\infty$.

\section{Discussion and conclusions}

We defined the Coriolis operator $\mathcal{C}$, which maps the space of continuously differentiable incompressible flows $\mathcal{V}^{1}(V)$ in a bounded region $V$ to itself. We showed that $\mathcal{C}$ is bounded with $\|\mathcal{C}\| \leqslant 1$ and $-\mathrm{i} \mathcal{C}$ is symmetric, thus establishing that the Coriolis modes are orthogonal with real frequencies $\omega,|\omega|<1$. We proved the polynomial Poisson-Neumann Theorem 6.2 for a sphere, thus showing that the space of incompressible polynomial flows $\mathcal{P}_{N}(V)$ of degree $\leqslant N$ in a sphere is invariant under $\mathcal{C}$. This and the self-adjointness of $-\mathrm{i} \mathcal{C}$ on $\mathcal{P}_{N}(V)$ enabled us to simply enumerate the Coriolis modes in a sphere and to establish their completeness, by invoking the Weierstrass approximation theorem and using Theorem 6.2 again. We 
also enumerated the modes directly from the known analytic solutions, with careful consideration given to the geostrophic modes.

The first approach, based on the polynomial Poisson-Neumann Theorem 6.2, is especially useful, since it does not require analytic expressions for the Coriolis modes. It will be shown elsewhere that Theorem 6.2 can be extended to spheroids and tri-axial ellipsoids, even if the rotation axis is tilted from the principal axes, and hence that the Coriolis modes are polynomials and complete in these cases. In fact, it has been known since Poincaré (1885a) (see also Hough 1895) that the inertial modes in a triaxial ellipsoid are polynomials in Cartesian coordinates. It will also be shown that the spaces of incompressible rotating periodic plane layer flows and rotating periodic duct flows can be decomposed using Fourier techniques into a sum of finite-dimensional spaces, which are invariant under the Coriolis operator $\mathcal{C}$. Consequently, the Coriolis modes are complete in periodic plane layer flows and a rotating periodic duct (Cui et al. 2014). Decomposition of incompressible flows in a rotating rectangular prism into finite-dimensional spaces invariant under the Coriolis operator fails.

Theorem 6.2 has no analogue in some simple geometries, which is consistent with these geometries having non-polynomial Coriolis modes. Thus the inertial pressure modes of a finite circular cylinder of radius $a$ rotating about its axis (Thomson 1880) are $P_{n, j}^{m}:=\mathbf{J}_{|m|}\left(\lambda_{|m|, j} s / a\right) \mathrm{e}^{\mathrm{i} m \phi} \cos n \pi z$, where $n \in \mathbb{N}, m \in \mathbb{Z}, \mathbf{J}_{|m|}$ is a Bessel function of the first kind and $\lambda_{|m|, j}$ is the $j$ th positive zero of $\lambda J_{|m|}^{\prime}(\lambda)-(m / \omega) \mathbf{J}_{|m|}(\lambda)$. The frequencies are given by $\omega^{2}=1 /\left[1+\left(\lambda_{|m|, j} / n \pi a\right)^{2}\right] \neq 0$. If $m=0$ and $\omega \rightarrow 0$ this condition reduces to $J_{0}^{\prime}(\lambda)=0$. Thus the geostrophic pressure modes are $P_{0, j}^{0}(s):=\mathrm{J}_{0}\left(\lambda_{0, j} s / a\right)$, where $\lambda_{0, j}$ is the $j$ th positive zero of $J_{0}^{\prime}(\lambda)=0$. The Coriolis modes are clearly not polynomials, specifically $\mathrm{J}_{|m|}(s) \mathrm{e}^{\mathrm{i} m \phi} \cos n \pi z$ is not polynomial. Thus it is not possible to construct the pressure $p$ in the Coriolis operator so that it maps $\mathcal{P}_{N}(V)$ to $\mathcal{P}_{N}(V)$, if $V$ is a cylinder, and Theorem 6.2 has no analogue in this case. Completeness of the discrete Coriolis modes for a cylinder is currently an open question; as the abstract completeness result outlined below indicates, it depends on the continuous spectrum.

A full analytical solution for the Coriolis modes in a spherical shell is not known. The difficulty with the separable solutions of the Poincaré's equation in the coordinate system (4.3) is that only one $\zeta$-surface is spherical, $\zeta=\omega$ corresponding to $s^{2}+z^{2}=$ 1. Theorem 6.1 for polynomial solutions of the Laplace-Neumann problem fails in a shell. Hence the existence Theorem 6.3 and the polynomial form of such modes also fails. Ray theory arguments suggest that the continuous spectrum is non-empty for a spherical shell (Israeli 1972; see also Stewartson 1972; Rieutord \& Valdettaro 1997). In fact, the existence of inertial modes in a spherical shell has been questioned (Stewartson \& Rickard 1969; Rieutord, Georgeot \& Valdettaro 2001).

We outline a general abstract existence argument for the Coriolis modes in a bounded volume with smooth boundaries. By a standard theorem in functional analysis the inner-product space $\mathcal{V}^{1}(V)$ can be completed to a Hilbert space $\overline{\mathcal{V}}^{1}(V)$, although the elements of the space may be difficult to characterise. The operator $\mathcal{C}$ can be extended to a bounded linear operator $\overline{\mathcal{C}}$ on $\overline{\mathcal{V}}^{1}(V)$, using continuity, and the operator $-\mathrm{i} \overline{\mathcal{C}}$ is therefore a bounded self-adjoint operator. It follows (Friedman 1982) that the residual spectrum $\sigma_{r}$ of $\mathcal{C}$ is empty and that the spectrum of $\mathcal{C}$, which is the union of the discrete and continuous spectrums, lies in $[-\mathrm{i}, \mathrm{i}]$. The spectral theorem for bounded self-adjoint operators (Friedman 1982, §6.7, Kreyszig 1978, §9.9 or Taylor \& Lay 1980, §6.6) applies to $-i \overline{\mathcal{C}}$ and implies that there exists a unique family of projection operators $\mathcal{E}_{\omega}$, which satisfy the conditions $\mathcal{E}_{\omega}=0$ if $\omega \leqslant-1$ 
and $\mathcal{E}_{\omega}=\boldsymbol{I}$ if $\omega>1$, such that $-\mathrm{i} \overline{\mathcal{C}}$ has the following representation in terms of a Riemann-Stieltjes integral (with a right upper limit following Friedman 1982),

$$
-\mathrm{i} \overline{\mathcal{C}}=\int_{-1}^{1+} \omega \mathrm{d} \mathcal{E}_{\omega} .
$$

Moreover, any $\boldsymbol{v} \in \overline{\mathcal{V}}^{1}(V)$ has the spectral resolution

$$
\boldsymbol{v}=\int_{-1}^{1+} \mathrm{d} \mathcal{E}_{\omega} \boldsymbol{v} .
$$

This is the general completeness result for the discrete and continuous modes of $\overline{\mathcal{C}}$. If the spectrum is purely discrete the Riemann-Stieltjes integral reduces to a sum over the eigenvalues of $-\mathrm{i} \overline{\mathcal{C}}$. The difficulty with the spectral theorem is that explicit construction of the spectral resolution is not possible except in special cases. Simple and practical characterisation of the spectral resolution is the subject of future work.

Spherical Coriolis modes are useful in applications and potentially even in computations, due to their explicit polynomial form and completeness. Spherical harmonic expansions of the pressure modes, toroidal-poloidal potentials and vector spherical harmonic expansions of the velocity modes will be given elsewhere. One application is to viscous solutions of the momentum equation, $\gamma \boldsymbol{v}+\mathcal{C} \boldsymbol{v}+E \boldsymbol{S} \boldsymbol{v}=\mathbf{0}$, or the viscous form of Poincaré's equation, and how the modes for $E=0$ approximate solutions in the limit $E \rightarrow 0$. Viscous asymptotic approximations have been given for spheres (Liao \& Zhang 2010b) and for a slender torus approximation to a spherical shell (Rieutord, Valdettaro \& Georgeot 2002). A second application is magnetohydrodynamic dynamos, including the kinematic dynamo action of Coriolis modes. It is known that plane inertial modes have maximal helicity, a property exploited by Moffatt (1970) to produce a mean-field dynamo alpha effect. In the spherical case simple spatial inertial modes are not mean-field dynamos at leading order, due to the absence of any Stokes drift (Herreman \& Lesaffre 2011), but simple linear combinations of spatial Coriolis modes do act as dynamos, such as polynomial Dudley-James flows (Dudley \& James 1989). The minimum magnetic Reynolds number $\min _{v \in \mathcal{P}_{N}(V)} R_{m}$ for dynamo action over polynomial flows of degree $N$ or less would be interesting, as would some analogue for Coriolis modes. A third application is forcing with feedback, such as in magnetohydromagnetic torsional oscillations, where the forcing depends on the magnetic field, which depends on the velocity via the magnetic induction equation.

Extensions of the Coriolis operator are possible. If the rotation is time-dependent, such as in precession, the incompressible Coriolis operator is time-dependent, $\mathcal{C}(t) \boldsymbol{v}=\boldsymbol{\Omega}(t) \times \boldsymbol{v}+\nabla p$, where $\boldsymbol{\nabla} \cdot \boldsymbol{v}=0$ in $V(t)$ and $\boldsymbol{n}(t) \cdot \boldsymbol{v}=0$ on $\partial V(t)$. A useful extension to fully compressible flows appears unlikely due to the variable density. Morton \& Shaughnessy (1972), Gans (1974) and Miles (1981) considered fully compressible inertial waves of an ideal gas in a cylinder rotating about its axis. Restricted compressible flows occur in various forms of the anelastic approximation, which all neglect the time dependence of the density in the equation for mass conservation (see Jones et al. 2011, §2). If $\rho^{\prime}$ and $p^{\prime}$ are the density and pressure perturbations of the basic state values $\bar{\rho}$ and $\bar{p}$ of a stably stratified gas shell modelling gaseous planets and stars, a generalisation of the Coriolis operator to this case (see Jones et al. 2011, Eq. (15)) is $\mathcal{C} v:=\widehat{\boldsymbol{\Omega}} \times \bar{\rho} \boldsymbol{v}+\bar{\rho} \boldsymbol{\nabla}\left(p^{\prime} / \bar{\rho}\right)$ with $\boldsymbol{\nabla} \cdot(\bar{\rho} \boldsymbol{v})=0$. The 
properties of this $\mathcal{C}$, which clearly depend on $\bar{\rho}$, are not pursued any further here. Further work is also required on anelastic approximations with $\nabla \cdot(\rho \boldsymbol{v})=0$, for which the Coriolis operator may be generalised to $\mathcal{C} \boldsymbol{u}:=\widehat{\boldsymbol{\Omega}} \times \boldsymbol{u}+\nabla p$, where $\boldsymbol{u}:=\rho \boldsymbol{v}$. This operator can be restricted to the space of polynomial momentum densities of degree $N$ or less, $\mathcal{P}_{N}(V)=\left\{\boldsymbol{u} \in \mathcal{P}_{N}^{3} \mid \nabla \cdot \boldsymbol{u}=0\right.$ in $V, \boldsymbol{n} \cdot \boldsymbol{u}=0$ on $\left.\partial V\right\}$ and the results for the incompressible operator would follow in this compressible case. An extension to moving boundaries, when $\boldsymbol{v}=\mathbf{0}$ is not a viscous solution in any rotating frame, is possible through linearisation. For example, consider a spherical shell with differentially rotating boundaries (Baruteau \& Rieutord 2013). The flow $\boldsymbol{v}=\mathbf{0}$ is a valid inviscid flow in any rotating reference frame concentric with the shell, but it is incompatible with a viscous solution. Let $\boldsymbol{v}_{0}$ satisfy

$$
\boldsymbol{v}_{0} \cdot \nabla \boldsymbol{v}_{0}+\widehat{\boldsymbol{\Omega}} \times \boldsymbol{v}_{0}=-\nabla p_{0}+E \nabla^{2} \boldsymbol{v}_{0}
$$

and $\boldsymbol{v}_{0}=\boldsymbol{v}_{\partial V}$, e.g. Taylor-Couette flow, and consider $\boldsymbol{v}^{\prime}:=\boldsymbol{v}-\boldsymbol{v}_{0}$. The linearised inviscid problem for $\boldsymbol{v}^{\prime}$ is $\partial_{t} \boldsymbol{v}^{\prime}+\left(\mathcal{C}+\mathcal{A}_{0}\right) \boldsymbol{v}^{\prime}=\boldsymbol{f}$, where the linearised advective operator $\mathcal{A}_{0} \boldsymbol{v}^{\prime}:=\boldsymbol{v}_{0} \cdot \boldsymbol{\nabla} \boldsymbol{v}^{\prime}+\nabla \boldsymbol{v}_{0}^{\mathrm{T}} \cdot \boldsymbol{v}^{\prime}$. It is easily shown that $\left(-\mathrm{i} \mathcal{A}_{0} \boldsymbol{v}_{1}^{\prime}, \boldsymbol{v}_{2}^{\prime}\right)=\left(\boldsymbol{v}_{1}^{\prime},-\mathrm{i} \mathcal{A}_{0} \boldsymbol{v}_{2}^{\prime}\right)$ and hence $-i \mathcal{A}_{0}$ is symmetric; but it is unbounded, unlike $\mathcal{C}$.

\section{Acknowledgements}

AJ is grateful for support through ERC Grant No. 247303 (MFECE). The authors are grateful to the anonymous referees for their constructive comments.

\section{Appendix Proof of Theorem 3.4}

We give an extension of Taylor's 1963 proof to Theorem 3.4.

Proof. Condition (3.33) is necessary, since integration of the $\phi$-component of (2.6) with $\lambda=0$ over $C(s)$ gives

$$
\int_{C(s)} f_{\phi} \mathrm{d} S=\int_{C(s)} \boldsymbol{v} \cdot \mathrm{d} \boldsymbol{S}=-\int_{S(s)} \boldsymbol{v} \cdot \mathrm{d} \boldsymbol{S}=-\int_{S(s)} g \mathrm{~d} S,
$$

noting that the total flux through the side $C(s)$ and ends $S(s)$ of the cylinder $V(s)$ is zero by $(3.32 c)$.

Condition (3.33) is also sufficient. Let $\boldsymbol{n}_{t}\left(\boldsymbol{n}_{b}\right)$ be the outward unit normal on the top (bottom) boundary $z=z_{t}\left(z=z_{b}\right)$. Then $v_{t}=v_{s} \sin \theta_{t}+v_{z} \cos \theta_{t}$ and $v_{b}=v_{s} \sin \theta_{b}-$ $v_{z} \cos \theta_{b}$, where $\cos \theta_{t}=\mathbf{1}_{z} \cdot \boldsymbol{n}_{t}$ and $\cos \theta_{b}=-\mathbf{1}_{z} \cdot \boldsymbol{n}_{b}$, and

$$
v_{s}=\frac{v_{t} \cos \theta_{b}+v_{b} \cos \theta_{t}}{\sin \left(\theta_{t}+\theta_{b}\right)}, \quad v_{z}=\frac{v_{t} \sin \theta_{b}-v_{b} \sin \theta_{t}}{\sin \left(\theta_{t}+\theta_{b}\right)} .
$$

Integration of the $\boldsymbol{n}_{t}$ and $\boldsymbol{n}_{b}$ components of (3.32a) yields

$$
v_{t}=g_{t}-\int_{z_{t}}^{z}(\nabla \times f)_{t} \mathrm{~d} z, \quad v_{b}=g_{b}-\int_{z_{b}}^{z}(\nabla \times f)_{b} \mathrm{~d} z .
$$

Transforming (A 2) to cylindrical polar coordinates yields

$$
v_{s}=v_{s}^{0}(\boldsymbol{f})+\frac{g_{t} \cos \theta_{b}+g_{b} \cos \theta_{t}}{\sin \left(\theta_{t}+\theta_{b}\right)}, \quad v_{z}=v_{z}^{0}(\boldsymbol{F})+\frac{g_{t} \sin \theta_{b}-g_{b} \sin \theta_{t}}{\sin \left(\theta_{t}+\theta_{b}\right)},
$$


where

$$
\begin{aligned}
v_{s}^{0}(\boldsymbol{f})= & -\left(\frac{\sin \theta_{t} \cos \theta_{b}}{\sin \left(\theta_{t}+\theta_{b}\right)} \int_{z_{t}}^{z}+\frac{\cos \theta_{t} \sin \theta_{b}}{\sin \left(\theta_{t}+\theta_{b}\right)} \int_{z_{b}}^{z}\right)(\nabla \times \boldsymbol{f})_{s} \mathrm{~d} z \\
& +\frac{\cos \theta_{t} \cos \theta_{b}}{\sin \left(\theta_{t}+\theta_{b}\right)} \int_{z_{b}}^{z_{t}}(\boldsymbol{\nabla} \times \boldsymbol{f})_{z} \mathrm{~d} z \\
v_{z}^{0}(\boldsymbol{f})= & -\left(\frac{\cos \theta_{t} \sin \theta_{b}}{\sin \left(\theta_{t}+\theta_{b}\right)} \int_{z_{t}}^{z}+\frac{\sin \theta_{t} \cos \theta_{b}}{\sin \left(\theta_{t}+\theta_{b}\right)} \int_{z_{b}}^{z}\right)(\nabla \times \boldsymbol{f})_{z} \mathrm{~d} z \\
& -\frac{\sin \theta_{t} \sin \theta_{b}}{\sin \left(\theta_{t}+\theta_{b}\right)} \int_{z_{b}}^{z_{t}}(\boldsymbol{\nabla} \times \boldsymbol{f})_{s} \mathrm{~d} z .
\end{aligned}
$$

The notation highlights the functional dependence of $v_{s}^{0}$ and $v_{z}^{0}$ on $f$. Both $v_{s}$ and $v_{z}$ are clearly unique, if $g$ is known.

The component $v_{\phi}$ must be determined by integrating (3.32b) and the $\phi$-component of (3.32a), namely

$$
\partial_{\phi} v_{\phi}=-\partial_{s}\left(s v_{s}\right)-s \partial_{z} v_{z}, \quad \partial_{z} v_{\phi}=\partial_{s} f_{z}-\partial_{z} f_{s},
$$

where $v_{s}$ and $v_{z}$ are given by (A 5) and (A 6). The equations (A 7) are integrable since the divergence of the right-hand side of $(3.32 a)$ is identically zero. When the $\phi$-integration in (A 7) is performed, a single-valued solution is obtained if and only if $\oint\left(\partial_{s}\left(s v_{s}\right)+s \partial_{z} v_{z}\right) \mathrm{d} \phi=0$. Substitution of the $z$-component of (3.32a) and (A 7) yields

$$
\begin{gathered}
\oint(\nabla \times \boldsymbol{f})_{s} \mathrm{~d} \phi=-\partial_{z} \oint f_{\phi} \mathrm{d} \phi, \quad \oint(\nabla \times \boldsymbol{f})_{z} \mathrm{~d} \phi=\frac{1}{s} \partial_{s} s \oint f_{\phi} \mathrm{d} \phi, \\
\partial_{s} \int_{C(s)} f_{\phi} \mathrm{d} S=\int_{z_{b}}^{z_{t}} \partial_{s} s \oint f_{\phi} \mathrm{d} \phi \mathrm{d} z+\left[s \oint f_{\phi} \mathrm{d} \phi \frac{\mathrm{d} z}{\mathrm{~d} s}\right]_{z_{b}}^{z_{t}},
\end{gathered}
$$

$\mathrm{d} z_{t} / \mathrm{d} s=-\tan \theta_{t}$ and $\mathrm{d} z_{b} / \mathrm{d} s=\tan \theta_{b}$ gives

$$
\oint\left(\partial_{s}\left(s v_{s}\right)+s \partial_{z} v_{z}\right) \mathrm{d} \phi=\partial_{s}\left(s \oint \frac{\cos \theta_{b} g_{t}+\cos \theta_{t} g_{b}}{\sin \left(\theta_{t}+\theta_{b}\right)} \mathrm{d} \phi+\frac{\cos \theta_{t} \cos \theta_{b}}{\sin \left(\theta_{t}+\theta_{b}\right)} \partial_{s} \int_{C(s)} f_{\phi} \mathrm{d} S\right) .
$$

The last expression vanishes since

$$
s \oint\left(\sec \theta_{t} g_{t}+\sec \theta_{b} g_{b}\right) \mathrm{d} \phi+\partial_{s} \int_{C(s)} f_{\phi} \mathrm{d} S=0
$$

which follows by differentiating (3.33) with respect to $s$, noting that $\mathrm{d} S=\sec \theta_{t} s \mathrm{~d} s \phi$ $\left(\mathrm{d} S=\sec \theta_{b} s \mathrm{~d} s \phi\right)$ on $z=z_{t}\left(z=z_{b}\right)$. Hence (3.33) is a necessary and sufficient condition for the single valuedness of $v_{\phi}$. The non-uniqueness of $v_{\phi}$ up to an arbitrary function of $s$ is obvious, as is the uniqueness from imposing condition (3.34).

\section{REFERENCES}

Baruteau, C. \& Rieutord, M. 2013 Inertial waves in a differentially rotating spherical shell. J. Fluid Mech. 719, 47-81. 
Bryan, G. H. 1889 The waves on a rotating liquid spheroid of finite ellipticity. Phil. Trans. R. Soc. Lond. A 180, 187-219.

Chossat, P. 1979 Bifurcation and stability of convective flows in a rotating or not rotating spherical shell. SIAM J. Appl. Maths 37, 624-647.

CUI, Z., ZhANG, K. \& LiAO, X. 2014 On the completeness of inertial wave modes in rotating annular channels. Geophys. Astrophys. Fluid Dyn. 108, 44-59.

DAVIS, P. J. 1975 Interpolation and Approximation. Dover.

Dormy, E., CARdin, P. \& JAUlT, D. 1998 MHD flow in a slightly differentially rotating spherical shell, with conducting inner core, in a dipolar magnetic field. Earth Planet. Sci. Lett. 160, $15-30$.

Dudley, M. L. \& JAmes, R. W. 1989 Time-dependent kinematic dynamos with stationary flows. Proc. R. Soc. Lond. A 425, 407-429.

Friedman, A. 1982 Foundations of Modern Analysis. Dover.

GANS, R. F. 1974 On the Poincaré problem for a compressible medium. J. Fluid Mech. 62, 657-675.

Greenspan, H. P. 1968 The Theory of Rotating Fluids. Cambridge University Press.

Herreman, W. \& Lesaffre, P. 2011 Stokes drift dynamos. J. Fluid Mech. 679, 32-57.

HIDE, R. 1995 The topographic torque on a bounding surface of a rotating gravitating fluid and the excitation by core motions of decadal fluctuations in the Earth's rotation. Geophys. Res. Lett. 22, 961-964.

Hollerbach, R. 1994a Magnetohydrodynamic Ekman and Stewartson layers in a rotating spherical shell. Proc. R. Soc. Lond. A 444, 333-346.

HollerbACH, R. $1994 b$ Imposing a magnetic field across a nonaxisymmetric shear layer in a rotating spherical shell. Phys. Fluids 6, 2540-2544.

Hollerbach, R. \& Proctor, M. R. E. 1993 Non-axisymmetric shear layers in a rotating spherical shell. In Theory of Solar and Planetary Dynamos (ed. P. C. Matthews, M. R. E. Proctor \& A. M. Rucklidge), pp. 145-152. Cambridge University Press.

Hough, S. S. 1895 The oscillations of a rotating ellipsoidal shell containing fluid. Phil. Trans. R. Soc. Lond. A 186, 469-506.

IsRAeLI, M. 1972 On trapped modes of rotating fluids in spherical shells. Stud. Appl. Maths 51, 219-237.

IVAnov, P. B. \& PAPAloizou, J. C. B. 2010 Inertial waves in rotating bodies: a WKBJ formalism for inertial modes and a comparison with numerical results. Mon. Not. R. Astron. Soc. 407, $1609-1630$.

Jones, C. A., Boronski, P., Brun, A. S., Glatzmaier, G. A., Gastine, T., Miesch, M. S. \& WICHT, J. 2011 Anelastic convection-driven dynamo benchmarks. Icarus 216, 120-135.

Kleeorin, N., Rogachevskit, I., Ruzmaikin, A., Soward, A. M. \& Starchenko, S. 1997 Axisymmetric flow between differentially rotating spheres in a dipole magnetic field. J. Fluid Mech. 344, 213-244.

KreYsZIG, E. 1978 Introductory Functional Analysis with Applications. John Wiley and Sons.

KUDLICK, M. D. 1966 On transient motions in a contained rotating fluid. PhD thesis.

LIAO, X. \& ZHANG, K. 2009 A new integral property of inertial waves in rotating fluid spheres. Proc. R. Soc. Lond. A 465, 1075-1091.

LiAO, X. \& ZhANG, K. 2010a A new Legendre-type polynomial and its application to geostrophic flow in rotating fluid spheres. Proc. R. Soc. Lond. A 466, 2203-2217.

LiAO, X. \& ZHANG, K. $2010 b$ Asymptotic and numerical solutions of the initial value problem in rotating planetary fluid cores. Geophys. J. Intl 180, 181-192.

Livermore, P. W. \& Hollerbach, R. 2012 Successive elimination of shear layers by a hierarchy of constraints in inviscid spherical-shell flows. J. Math. Phys. 53, 073104.

MacRobert, T. M. 1947 Spherical Harmonics An Elementary Treatise on Harmonic Functions with Applications, 2nd edn. Methuen.

Maxwell, J. C. 1891 A Treatise on Electricity and Magnetism, 3rd edn. Clarendon.

Miles, J. W. 1981 Waves in a rapidly rotating gas. J. Fluid Mech. 107, 487-497.

MoffatT, H. K. 1970 Dynamo action associated with random inertial waves in a rotating conducting fluid. J. Fluid Mech. 44, 705-719. 
Morton, J. B. \& Shaughnessy, E. J. 1972 Waves in a gas in solid-body rotation. J. Fluid Mech. 56, 277-286.

Nurijanyan, S., Bokhove, O. \& MaAs, L. R. M. 2013 A new semi-analytical solution for inertial waves in a rectangular parallelepiped. Phys. Fluids 25, 126601.

PAZY, A. 1983 Semigroups of Linear Operators and Applications to Partial Differential Equations. Springer.

Pedlosky, J. \& Greenspan, H. P. 1967 A simple laboratory model for the oceanic circulation. J. Fluid Mech. 27, 291-304.

PoInCARÉ, H. $1885 a$ Sur l'équilibre d'une masse fluide animée d'un mouvement de rotation. Acta Mathematica 7, 259-380.

PoINCARÉ, H. $1885 b$ Sur l'équilibre d'une masse fluide animée d'un mouvement de rotation. Bull. Astron. I 2, 109-118.

PoINCARÉ, H. 1885c Sur l'équilibre d'une masse fluide animée d'un mouvement de rotation. Bull. Astron. I 2, 405-413.

RAO, D. B. 1966 Free gravitational oscillations in rotating rectangular basins. J. Fluid Mech. 25, 523-555.

RIEUTORD, M. 1991 Linear theory of rotating fluids using spherical harmonics part II, time periodic flows. Geophys. Astrophys. Fluid Dyn. 59, 185-208.

Rieutord, M., Georgeot, B.\& VAldettaro, L. 2001 Inertial waves in a rotating spherical shell: attractors and asymptotic spectrum. J. Fluid Mech. 435, 103-144.

Rieutord, M. \& Valdettaro, L. 1997 Inertial waves in a rotating spherical shell. J. Fluid Mech. 341, 77-99.

Rieutord, M., Valdettaro, L. \& Georgeot, B. 2002 Analysis of singular inertial modes in a spherical shell: the slender toroidal shell model. J. Fluid Mech. 463, 345-360.

Schechter, M. 1971 Principles of Functional Analysis. Academic.

SOWARD, A. M. \& Hollerbach, R. 2000 Non-axisymmetric magnetohydrodynamic shear layers in a rotating spherical shell. J. Fluid Mech. 408, 239-274.

STEWARTSON, K. 1972 On trapped oscillations of a rotating fluid in a thin spherical shell II. Tellus 24, 283-287.

Stewartson, K. \& Rickard, J. A. 1969 Pathological oscillations of a rotating fluid. J. Fluid Mech. 35, 759-773.

TAYLOR, G. I. 1922 Tidal oscillations in gulfs and rectangular basins. Proc. Lond. Math. Soc. 20, $148-181$.

TAYLOR, J. B. 1963 The magneto-hydrodynamics of a rotating fluid and the Earth's dynamo problem. Proc. R. Soc. Lond. A 274, 274-283.

TAYlor, A. E. \& LAY, D. C. 1980 Introduction to Functional Analysis, 2nd edn. John Wiley and Sons.

Thomson, W. 1877 On the precessional motion of a liquid. Nature 15 (379), 289-308.

Thomson, W. 1880 Vibrations of a columnar vortex. Phil. Mag. 10, 155-168.

Trefethen, L. N. 1997 Pseudospectra of linear operators. SIAM Rev. 39, 383-406.

ZHANG, K. 1993 On equatorially trapped boundary inertial waves. J. Fluid Mech. 248, 203-217.

Zhang, K., Earnshaw, P., Liao, X. \& Busse, F. H. 2001 On inertial waves in a rotating fluid sphere. J. Fluid Mech. 437, 103-119.

Zhang, K., LiaO, X. \& EARnshaw, P. 2004 The Poincaré equation: a new polynomial and its unusual properties. J. Math. Phys. 45, 4777-4790. 